Pacific

Journal of

Mathematics

\title{
COHOMOLOGIE DE CHEVALLEY DES GRAPHES VECTORIELS
}

Walid Aloulou, Didier Arnal et Ridha Chatbouri 


\title{
COHOMOLOGIE DE CHEVALLEY DES GRAPHES VECTORIELS
}

\author{
Walid Aloulou, Didier Arnal et Ridha Chatbouri
}

\begin{abstract}
The space of smooth functions and vector fields on $\mathbb{R}^{d}$ is a Lie subalgebra of the (graded) Lie algebra $T_{\text {poly }}\left(\mathbb{R}^{d}\right)$, equipped with the Scouten bracket. Here we compute the cohomology of this subalgebra for the adjoint representation in $T_{\text {poly }}\left(\mathbb{R}^{d}\right)$, restricting ourselves to the case of cochains defined with purely aerial Kontsevich graphs, as in Arnal, Gammella and Masmoudi, Pacific Journal of Mathematics 218 (2005), 201-239.

We find results very similar to the classical result of Gelfand-Fuks and those of De Wilde-Lecomte.
\end{abstract}

L'espace des champs de vecteurs augmenté des fonctions $C^{\infty}$ sur $\mathbb{R}^{d}$ est une sous algèbre de Lie de l'algèbre de Lie (graduée) de l'espace $T_{\text {poly }}\left(\mathbb{R}^{d}\right)$ des champs de tenseurs sur $\mathbb{R}^{d}$ muni du crochet de Schouten.

On calcule ici la cohomologie des représentations adjointes de cette sous algèbre de Lie, en se restreignant à des cochaînes définies par des graphes de Kontsevich aériens comme dans [Arnal et al. 2005]. On retrouve les résultats bien connus de Gelfand et Fuks [1970] et De Wilde et Lecomte [1983].

\section{Introduction}

Notons $T_{\text {poly }}\left(\mathbb{R}^{d}\right)$ l'espace des tenseurs contravariants complètement antisymétriques sur $\mathbb{R}^{d}$. Cet espace, muni du crochet de Schouten et de la graduation

$$
\operatorname{deg}(\alpha)=k-1 \quad \text { si } \alpha \text { est un } k \text {-tenseur, }
$$

est une algèbre de Lie graduée.

Cette algèbre de Lie contient une sous-algèbre de Lie intéressante : $\operatorname{Vect}\left(\mathbb{R}^{d}\right)$, espace des tenseurs de degré négatif ou nul, c'est à dire l'algèbre de Lie des champs

MSC2000: primary 17B56; secondary 53D55, 05 C99.

Mots-clefs: formality, vector graph, Chevalley cohomology.

Ce travail a été effectué dans le cadre de l'accord CMCU 03 S 1502. W. Aloulou et R. Chatbouri remercient l'Université de Bourgogne pour l'accueil dont ils ont bénéficié au cours de leurs séjours, D. Arnal remercie la Faculté des Sciences de Monastir pour l'accueil dont il a bénéficié au cours de ses séjours. 
de vecteurs $\xi$ augmentée de l'espace des fonctions $f$ de $C^{\infty}$ muni du crochet usuel des champs de vecteurs étendu aux fonctions par :

$$
[\xi, f]=-[f, \xi]=\xi f \quad \text { et } \quad\left[f_{1}, f_{2}\right]=0 .
$$

La cohomologie de Chevalley des champs de vecteurs a été calculée par plusieurs auteurs. En particulier dans [De Wilde et Lecomte 1983], la cohomologie à valeurs dans les $q$-formes sur une variété $M$ est déterminée explicitement. Elle dépend de la cohomologie de De Rham sur $M$ et elle contient une partie "universelle":

Dans le cas de $\mathbb{R}^{d}$, cette cohomologie n'est pas triviale, elle est isomorphe à $\bigwedge_{\text {inv }}(\mathfrak{g l}(d, \mathbb{R}))$ l'espace des formes multilinéaires alternées sur $\mathfrak{g l}(d, \mathbb{R})$ invariantes par la représentation adjointe. Une base de cet espace est donnée $\operatorname{par} \zeta^{\left(j_{1}\right)} \wedge \cdots \wedge \zeta^{\left(j_{q}\right)}$ où $j_{k}$ est impair, $j_{1}<\cdots<j_{q}<2 d$ et $\zeta^{(j)}$ est l'antisymétisé de l'application trace : $\zeta^{(j)}\left(A_{1}, \ldots, A_{j}\right)=\mathfrak{a}\left(\operatorname{tr}\left(A_{1} \ldots A_{j}\right)\right)$.

L'isomorphisme est donné par les $\theta^{(j)}\left(\xi_{1}, \ldots, \xi_{j}\right)=\zeta^{(j)}\left(\operatorname{Jac}\left(\xi_{1}\right), \ldots, \operatorname{Jac}\left(\xi_{j}\right)\right)$ où les $\left(\xi_{i}\right)_{i}$ sont des champs de vecteurs sur $\mathbb{R}^{d}$ et $\operatorname{Jac}\left(\xi_{i}\right)$ est la matrice jacobienne de $\xi_{i}$.

D'autre part, pour construire une formalité sur $\mathbb{R}^{d}$, M. Kontsevich a défini des cochaînes $\sum a_{\Gamma, \mathcal{O}} B_{\Gamma, \mathcal{O}}$ de $T_{\text {poly }}\left(\mathbb{R}^{d}\right)$ à valeurs dans les opérateurs multidifférentiels $D_{\text {poly }}\left(\mathbb{R}^{d}\right)$ à l'aide des graphes $\Gamma$ orientés par un ordre $\mathcal{O}$ sur ses arêtes (voir le paragraphe 3 pour une définition explicite). Le problème de la construction d'une formalité sur $\mathbb{R}^{d}$ fait apparaître diverses cohomologies : celle de Hochschild a été calculée dans [Arnal et Masmoudi 2002], la cohomologie de Chevalley-Harrison est nulle d'après [Gammella et Halbout 2003] et celle de Chevalley reste inconnue.

On étudie alors l'espace des cochaînes $\sum a_{\Gamma, \bigcirc} B_{\Gamma, \bigcirc}$ définies par des combinaisons linéaires symétriques de graphes ayant une propriété de symétrie naturelle. Dans [Arnal et al. 2005], il est montré que ces cochaînes ne dépendent que de la partie "aérienne" $\Delta$ d'un graphe $\Gamma$. La cohomologie de Chevalley se réduit à celle du module $T_{\text {poly }}^{0}\left(\mathbb{R}^{d}\right)=C^{\infty}\left(\mathbb{R}^{d}\right)$ pour chaque $d \in \mathbb{N}^{*}$. L'opérateur de cohomologie peut alors être défini sur l'espace des graphes, il correspond à une suite d' "éclatement" des sommets.

Dans cet article, on se restreint au cas de $\operatorname{Vect}\left(\mathbb{R}^{d}\right)$. Le problème est alors proche de celui étudié par De Wilde et Lecomte restreint aux 0-formes.

Cependant, comme on veut travailler uniquement sur des cochaînes construites par des graphes vectoriels où au plus une arête part d'un sommet de chacun de ces graphes, il nous faut reprendre et adapter complètement leur preuve. La définition du symbole d'une cochaîne doit tenir compte des 0 -tenseurs de $\operatorname{Vect}\left(\mathbb{R}^{d}\right)$. La contraction (ou homotopie) peut être définie directement sur les graphes vectoriels. On en déduit que la cohomologie de Chevalley de ce complexe est donnée par 
les combinaisons linéaires à coefficients constants des "roues impaires" $R_{j}$. Ces graphes $R_{j}$ sont tels que $B_{R_{j}}=\theta^{(j)}$.

On retrouve donc le même résultat que celui de [De Wilde et Lecomte 1983], ce qui prouve le caractère universel de ces cochaînes.

\section{Notations et définitions}

On considère l'espace $T_{\text {poly }}\left(\mathbb{R}^{d}\right)$ des tenseurs contravariants complètement antisymétriques sur $\mathbb{R}^{d}$. Si $\mathscr{X}$ désigne l'espace des champs de vecteurs $\xi$ sur $\mathbb{R}^{d}$, on construit Tens $\left(\mathbb{R}^{d}\right)$ comme l'algèbre associative libre sur $C^{\infty}\left(\mathbb{R}^{d}\right)$ engendrée par les champs constants $\partial_{1}, \ldots, \partial_{d}$. Alors $T_{\text {poly }}\left(\mathbb{R}^{d}\right)$ est le quotient de Tens $\left(\mathbb{R}^{d}\right)$ par l'idéal engendré par $\{\xi \otimes \eta-\eta \otimes \xi: \xi \in \mathscr{X}, \eta \in \mathscr{X}\}$. Cet espace est muni d'un produit associatif $\wedge$, tout élément de $T_{\text {poly }}\left(\mathbb{R}^{d}\right)$ est une somme de produits de la forme $\xi_{1} \wedge \cdots \wedge \xi_{k}$ et de fonctions $f$. On peut aussi écrire tout tenseur $\alpha$ de $T_{\text {poly }}\left(\mathbb{R}^{d}\right)$ de façon unique sous la forme

$$
\alpha=\sum_{k=0}^{K} \sum_{i_{1}, \ldots, i_{k}} \alpha_{(k)}^{i_{1} \ldots i_{k}} \partial_{i_{1}} \wedge \cdots \wedge \partial_{i_{k}}
$$

(On prend la convention que les coordonnées $\alpha_{(k)}^{i_{1} \ldots i_{k}}$ sont des fonctions $C^{\infty}$ et sont complètement antisymétriques en $i_{1}, \ldots, i_{k}$.) On a donc aussi

$$
\alpha=\sum_{k=0}^{K} \sum_{i_{1}<\cdots<i_{k}} k ! \alpha_{(k)}^{i_{1} \ldots i_{k}} \partial_{i_{1}} \wedge \cdots \wedge \partial_{i_{k}}
$$

On place sur $\mathbb{R}^{d}$ la connexion plate triviale $\nabla$, c'est à dire la connexion pour la structure riemannienne usuelle de $\mathbb{R}^{d}$. On a donc

$$
\nabla_{\xi} f=\xi f, \quad \nabla_{\xi} \eta=\nabla_{\xi}\left(\sum_{i} \eta^{i} \partial_{i}\right)=\sum_{i}\left(\xi \eta^{i}\right) \partial_{i} \quad\left(f \in C^{\infty}\left(\mathbb{R}^{d}\right), \xi, \eta \in \mathscr{X}\right) .
$$

Il y a un prolongement unique de $\nabla_{\xi}$ en une dérivation de $T_{\text {poly }}\left(\mathbb{R}^{d}\right)$. On impose

$$
\nabla_{\xi}(\alpha \wedge \beta)=\left(\nabla_{\xi} \alpha\right) \wedge \beta+\alpha \wedge\left(\nabla_{\xi} \beta\right),
$$

on obtient une solution et une seule définie par

$$
\nabla_{\xi}\left(\eta_{1} \wedge \cdots \wedge \eta_{\ell}\right)=\sum_{j=1}^{\ell}(-1)^{j-1}\left(\nabla_{\xi} \eta_{j}\right) \wedge \eta_{1} \wedge \ldots \widehat{\eta}_{j} \cdots \wedge \eta_{\ell}
$$

ou par

$$
\nabla_{\xi} \alpha=\sum_{i_{1}, \ldots, i_{k}}\left(\xi \alpha^{i_{1} \ldots i_{k}}\right) \partial_{i_{1}} \wedge \cdots \wedge \partial_{i_{k}}
$$


Maintenant, on considère $T_{\text {poly }}\left(\mathbb{R}^{d}\right)$ comme une algèbre graduée par $|\alpha|=k$ si $\alpha$ est un $k$-tenseur et on utilise systématiquement la règle de Koszul. Si $\xi \in \mathscr{X}, \nabla_{\xi}$ est une dérivation de degré 0 , le produit $\wedge$ est aussi de degré 0 , les formules ci-dessus sont donc cohérentes avec cette règle. Cependant l'application

$$
\nabla: \xi \mapsto \nabla_{\xi}
$$

est maintenant homogène de degré -1 de $\mathscr{X}$ vers l'espace $\operatorname{Der}\left(T_{\text {poly }}\left(\mathbb{R}^{d}\right)\right)$ des dérivations de $T_{\text {poly }}\left(\mathbb{R}^{d}\right)$. On veut la prolonger comme une dérivation.

On cherche donc $\nabla: T_{\text {poly }}\left(\mathbb{R}^{d}\right) \rightarrow \operatorname{Der}\left(T_{\text {poly }}\left(\mathbb{R}^{d}\right)\right)$ qui la prolonge et telle que

$$
\nabla_{\alpha \wedge \beta}(\gamma)=(-1)^{|\alpha|} \alpha \wedge\left(\nabla_{\beta} \gamma\right)+(-1)^{|\beta||\gamma|}\left(\nabla_{\alpha} \gamma\right) \wedge \beta
$$

Lemme 2.1 (Le prolongement). Un tel prolongement existe et il est unique, il est défini par $\nabla_{f}=0$ si $f \in C^{\infty}\left(\mathbb{R}^{d}\right)$ et soit

$$
\begin{aligned}
\nabla_{\xi_{1} \wedge \cdots \wedge \xi_{k}}\left(\eta_{1} \wedge\right. & \left.\cdots \wedge \eta_{\ell}\right) \\
& =\sum_{i=1}^{k} \sum_{j=1}^{\ell}(-1)^{i+j} \xi_{1} \wedge \cdots \widehat{\xi}_{i} \cdots \wedge \xi_{k} \wedge\left(\nabla_{\xi_{i}} \eta_{j}\right) \wedge \eta_{1} \wedge \cdots \widehat{\eta}_{j} \cdots \wedge \eta_{\ell}
\end{aligned}
$$

soit

$$
\nabla_{\alpha} \beta=\sum_{r=1}^{k}(-1)^{r-1} \sum_{\substack{i_{1}, \ldots, i_{k} \\ j_{1}, \ldots, j_{\ell}}} \alpha^{i_{1} \ldots i_{k}}\left(\partial_{i_{r}} \beta^{j_{1} \ldots j_{\ell}}\right) \partial_{i_{1}} \wedge \cdots \widehat{\partial_{i_{r}}} \cdots \wedge \partial_{i_{k}} \wedge \partial_{j_{1}} \wedge \cdots \wedge \partial_{j_{\ell}} .
$$

Remarquons que l'on retrouve l'opération notée • dans [Arnal et al. 2002] :

$$
\nabla_{\alpha} \beta=\alpha \bullet \beta .
$$

Démonstration. Soit $\xi$ un champ de vecteurs et $f \in C^{\infty}\left(\mathbb{R}^{d}\right)$. On doit avoir $\nabla_{(f \xi)}=$ $\nabla_{f \wedge \xi}$ ou, pour tout $k$-tenseur $\alpha$,

$$
f \nabla_{\xi} \alpha=\nabla_{(f \xi)}(\alpha)=f\left(\nabla_{\xi} \alpha\right)+(-1)^{k}\left(\nabla_{f} \alpha\right) \wedge \xi .
$$

$\nabla_{f} \alpha$ est un $k-1$-tenseur. S'il n'est pas nul, on peut choisir $\xi$ tel que $\left(\nabla_{f} \alpha\right) \wedge \xi \neq 0$, ce qui est absurde.

On montre ensuite par récurrence sur $k$ que $\nabla_{\xi_{1} \wedge \cdots \wedge \xi_{k}}\left(\eta_{1} \wedge \cdots \wedge \eta_{\ell}\right)$ ne peut être que ce qui est annoncé. Enfin que l'application $\nabla$ ainsi définie a bien les propriétés demandées. La formule donnant $\nabla_{\alpha} \beta$ est une conséquence immédiate de la première formule.

Maintenant on a par construction

$$
\nabla_{\xi} \eta-\nabla_{\eta} \xi=[\xi, \eta] \quad(\xi, \eta \in \mathscr{X}) .
$$

Traditionnellement, pour étendre le crochet des champs de vecteurs en le crochet de Schouten, défini sur $T_{\text {poly }}\left(\mathbb{R}^{d}\right)$, on choisit la graduation $\operatorname{deg}(\alpha)=k-1$ si $\alpha$ est 
un $k$-tenseur, le crochet des champs de vecteurs est antisymétrique, de degré 0 , et il se prolonge d'une façon unique en un crochet antisymétrique toujours de degré 0 sur $T_{\text {poly }}\left(\mathbb{R}^{d}\right)$ qui est une dérivation “à droite", c'est à dire en un crochet tel que

$$
\begin{aligned}
{[\alpha, \beta \wedge \gamma] } & =[\alpha, \beta] \wedge \gamma+(-1)^{(\operatorname{deg}(\beta)-1) \operatorname{deg}(\alpha)} \beta \wedge[\alpha, \gamma] \\
{[\beta, \alpha] } & =-(-1)^{\operatorname{deg}(\alpha) \operatorname{deg}(\beta)}[\alpha, \beta]
\end{aligned}
$$

(en effet, $\operatorname{deg}(\wedge)=-1$ maintenant). Ce prolongement unique est donné par $[f, g]=$ 0 si $f$ et $g$ sont des fonctions, $\operatorname{par}[\xi, f]=-[f, \xi]=\xi f$ si $\xi$ est un champ de vecteurs et $f$ une fonction et par

$\left[\xi_{1} \wedge \cdots \wedge \xi_{k}, \eta_{1} \wedge \cdots \wedge \eta_{\ell}\right]$

$$
=\sum_{i=1}^{k} \sum_{j=1}^{\ell}(-1)^{k-i+j-1} \xi_{1} \wedge \cdots \widehat{\xi}_{i} \cdots \wedge \xi_{k} \wedge\left[\xi_{i}, \eta_{j}\right] \wedge \eta_{1} \wedge \cdots \widehat{\eta}_{j} \cdots \wedge \eta_{\ell} .
$$

Il vérifie la relation

$$
[\alpha \wedge \beta, \gamma]=\alpha \wedge[\beta, \gamma]+(-1)^{(\operatorname{deg}(\beta)+1) \operatorname{deg}(\gamma)}[\alpha, \gamma] \wedge \beta
$$

Avec nos notations, on peut aussi définir le crochet de Schouten par :

$$
[\alpha, \beta]=(-1)^{\operatorname{deg}(\alpha)} \nabla_{\alpha} \beta-(-1)^{(\operatorname{deg}(\alpha)+1) \operatorname{deg}(\beta)} \nabla_{\beta} \alpha .
$$

Cependant, nous gardons ici la graduation $|\alpha|=k$ si $\alpha$ est un $k$-tenseur. Le crochet des champs de vecteurs devient un produit commutatif. On le prolonge comme dans [Kontsevich 2003] en une opération $Q$ symétrique de degré - 1 vérifiant

$$
\begin{aligned}
Q(\alpha, \beta \wedge \gamma) & =Q(\alpha, \beta) \wedge \gamma+(-1)^{|\beta|(|\alpha|-1)} \beta \wedge Q(\alpha, \gamma) \\
Q(\beta, \alpha) & =(-1)^{|\alpha||\beta|} Q(\alpha, \beta) .
\end{aligned}
$$

Lemme 2.2 (L'opérateur $Q$ ). Il y a un prolongement et un seul $Q$ du crochet des champs de vecteurs vérifiant ces relations. Ce prolongement est donné par

$$
Q(\alpha, \beta)=\nabla_{\alpha} \beta+(-1)^{|\alpha||\beta|} \nabla_{\beta} \alpha .
$$

Il vérifie

$$
\begin{gathered}
Q(\alpha \wedge \beta, \gamma)=(-1)^{|\alpha|} \alpha \wedge Q(\beta, \gamma)+(-1)^{|\beta \| \gamma|} Q(\alpha, \gamma) \wedge \beta \\
Q(\alpha, \beta)=\sum_{\substack{i_{1} \ldots i_{k} \\
j_{1} \ldots j_{\ell}}}\left(\sum_{r=1}^{k}(-1)^{r-1} \alpha^{i_{1} \ldots i_{k}}\left(\partial_{i_{r}} \beta^{j_{1} \ldots j_{\ell}}\right) \partial_{i_{1}} \wedge \cdots \widehat{\partial_{i_{r}}} \cdots \wedge \partial_{i_{k}} \wedge \partial_{j_{1}} \wedge \cdots \wedge \partial_{j_{\ell}}\right. \\
\left.\quad+\sum_{s=1}^{\ell}(-1)^{k+s} \beta^{j_{1} \ldots j_{\ell}}\left(\partial_{j_{s}} \alpha^{i_{1} \ldots i_{k}}\right) \partial_{i_{1}} \wedge \cdots \wedge \partial_{i_{k}} \wedge \partial_{j_{1}} \wedge \cdots \widehat{\partial_{j_{s}}} \cdots \wedge \partial_{j_{\ell}}\right)
\end{gathered}
$$

et $[\alpha, \beta]=(-1)^{\operatorname{deg}(\alpha)} Q(\alpha, \beta)$. 
Démonstration. Supposons que $Q$ soit une telle extension. $Q$ étant de degré -1 , on a $Q(f, g)=0$ si $f$ et $g$ sont des fonctions. Maintenant

$$
Q(\xi, f \eta)=Q(\xi, f) \eta+f Q(\xi, \eta)=[\xi, f \eta]=(\xi f) \eta+f[\xi, \eta],
$$

donc $Q(\xi, f)=Q(f, \xi)=\xi f=\nabla_{\xi} f+\nabla_{f} \xi$. L'application $\beta \mapsto Q(\xi, \beta)$ étant une dérivation, on montre par récurrence sur $\ell$ que

$$
Q\left(\xi, \eta_{1} \wedge \cdots \wedge \eta_{\ell}\right)=\sum_{j=1}^{\ell}(-1)^{j-1}\left[\xi, \eta_{j}\right] \wedge \eta_{1} \wedge \cdots \widehat{\eta}_{j} \cdots \wedge \eta_{\ell}
$$

Par récurrence sur $k$, on montre ensuite que

$$
\begin{aligned}
& Q\left(\xi_{1} \wedge \cdots \wedge \xi_{k}, \eta_{1} \wedge \cdots \wedge \eta_{\ell}\right) \\
& \quad=\sum_{\substack{i=1, \ldots, k \\
j=1, \ldots, \ell}}(-1)^{i+j} \xi_{1} \wedge \cdots \widehat{\xi}_{i} \cdots \wedge \xi_{k} \wedge\left[\xi_{i}, \eta_{j}\right] \wedge \eta_{1} \wedge \cdots \widehat{\eta}_{j} \cdots \wedge \eta_{\ell}
\end{aligned}
$$

Ceci nous dit que si $Q$ existe, elle est unique et que c'est

$$
Q(\alpha, \beta)=\nabla_{\alpha} \beta+(-1)^{|\alpha||\beta|} \nabla_{\beta} \alpha .
$$

Maintenant les propriétés de $\nabla$ montrent que cette formule définit bien une bidérivation symétrique de degré -1 , extension du crochet des champs de vecteurs. Les dernières formules du lemme sont immédiates.

Reprenons maintenant le choix de signes donné dans [Arnal et al. 2002]. Soit $\sigma$ une permutation de $\{1, \ldots, n\}$. On note $\varepsilon(\sigma)$ sa signature. Si $v_{1}, \ldots, v_{n}$ sont $n$ vecteurs homogènes d'un espace vectoriel $V$ gradué par deg, on note $\varepsilon_{\operatorname{deg}(v)}(\sigma)$ la signature de la permutation que $\sigma$ induit sur les $v$ de degrés impairs. Par construction ces signatures sont des homomorphismes de groupe. Si $C$ est une application $n$-linéaire sur $V^{n}$, à valeurs dans un espace vectoriel, on dira que $C$ est symétrique (resp. antisymétrique) si pour toute permutation $\sigma \in S_{n}$ et tout $v_{1}, \ldots, v_{n}$ homogènes,

$$
C\left(v_{\sigma(1)}, \ldots, v_{\sigma(n)}\right)=\varepsilon_{\operatorname{deg}(v)}(\sigma) C\left(v_{1}, \ldots, v_{n}\right)
$$

(respectivement $\left.C\left(v_{\sigma(1)}, \ldots, v_{\sigma(n)}\right)=\varepsilon(\sigma) \varepsilon_{\operatorname{deg}(v)}(\sigma) C\left(v_{1}, \ldots, v_{n}\right)\right)$.

Ceci est équivalent à

$$
\begin{gathered}
C\left(v_{1}, \ldots, v_{i+1}, v_{i}, \ldots, v_{n}\right)=(-1)^{\operatorname{deg}\left(v_{i}\right) \operatorname{deg}\left(v_{i+1}\right)} C\left(v_{1}, \ldots, v_{n}\right) \\
\left(\text { resp. } C\left(v_{1}, \ldots, v_{i+1}, v_{i}, \ldots, v_{n}\right)=-(-1)^{\operatorname{deg}\left(v_{i}\right) \operatorname{deg}\left(v_{i+1}\right)} C\left(v_{1}, \ldots, v_{n}\right)\right)
\end{gathered}
$$

pour tout $i$.

Changeons de graduation sur $V$ et posons $|v|=\operatorname{deg}(v)+1$. Posons

$$
\eta_{v}(\sigma)=\varepsilon_{\operatorname{deg}(v)}(\sigma) \varepsilon_{|v|}(\sigma) \varepsilon(\sigma) .
$$


Si nous nous donnons une application $\tau$ de $V^{n}$ dans $\{ \pm 1\}$ telle que pour tout $\sigma$ et tout $v_{1}, \ldots, v_{n}$,

$$
\tau\left(v_{\sigma(1)}, \ldots, v_{\sigma(n)}\right)=\eta_{v}(\sigma) \tau\left(v_{1}, \ldots, v_{n}\right),
$$

alors on peut faire correspondre à toute application $n$-linéaire deg-antisymétrique $C^{\prime}$ une application $n$-linéaire | |-symétrique $C$ en posant $C=\tau C^{\prime}$ ou

$$
C\left(v_{1}, \ldots, v_{n}\right)=\tau\left(v_{1}, \ldots, v_{n}\right) C^{\prime}\left(v_{1}, \ldots, v_{n}\right) .
$$

Un tel choix a été fait dans [Arnal et al. 2002] où on a posé $\tau\left(v_{1}, \ldots, v_{n}\right)=\eta_{v}(\sigma)$ où $\sigma$ est la permutation rangeant les $v_{i}$ deg-pairs au début sans changer leur ordre et les $v_{j}$ deg-impairs en fin sans changer leur ordre.

Du fait de la relation établie dans le lemme 2.2 entre [, ] et $Q$, nous posons ici

$$
\tau\left(\alpha_{1}, \ldots, \alpha_{n}\right)=(-1)^{\sum_{i=1}^{n}(n-i) \operatorname{deg}\left(\alpha_{i}\right)} .
$$

En fait (2-1) est vrai pour $\sigma=(i, i+1)$ donc pour tout $\sigma$ puisque chaque membre de (2-1) définit une action de $S_{n}$. On a donc

$$
Q=\tau[,] .
$$

Définition 2.3 (Cohomologie de Chevalley). Soit $C^{\prime}$ une $n$-cochaîne, c'est à dire une application $n$-linéaire deg-antisymétrique de $\left(T_{\text {poly }}\left(\mathbb{R}^{d}\right)\right)^{n}$ dans $T_{\text {poly }}\left(\mathbb{R}^{d}\right)$, homogène de degré $\operatorname{deg}\left(C^{\prime}\right)$. Le cobord $\partial^{\prime} C^{\prime}$ de $C^{\prime}$ est par définition l'application $(n+1)$-linéaire deg-antisymétrique

$$
\begin{aligned}
& \left(\partial^{\prime} C^{\prime}\right)\left(\alpha_{0}, \ldots, \alpha_{n}\right) \\
& =\sum_{i=0}^{n}(-1)^{i} \varepsilon_{\operatorname{deg} \alpha}(i, 0 \ldots \hat{\imath} \ldots n)(-1)^{\operatorname{deg} C^{\prime} \operatorname{deg} \alpha_{i}}\left[\alpha_{i}, C^{\prime}\left(\alpha_{0}, \ldots \widehat{\alpha}_{i} \ldots, \alpha_{n}\right)\right] \\
& \quad-\frac{1}{2} \sum_{i \neq j} \varepsilon_{\operatorname{deg} \alpha}(i, j, 0, \ldots \hat{\imath} \ldots \hat{\jmath} \ldots n)(-1)^{i+j-1} C^{\prime}\left(\left[\alpha_{i}, \alpha_{j}\right], \alpha_{0}, \ldots \widehat{\alpha}_{i} \ldots \widehat{\alpha}_{j} \ldots, \alpha_{n}\right) .
\end{aligned}
$$

Une $n$-cochaîne $C^{\prime}$ telle que $\partial^{\prime} C^{\prime}=0$ est appelée un cocycle, une $n$-cochaîne de la forme $C^{\prime}=\partial^{\prime} A^{\prime}$ est appelée un cobord.

Remarque 2.4. La restriction de $\partial^{\prime}$ à l'espace des $n$-cochaînes $C^{\prime}$ deg-antisymétriques définies seulement sur les champs de vecteurs et à valeurs dans les fonctions

$$
C^{\prime}:\left(\mathscr{X}\left(\mathbb{R}^{d}\right)\right)^{n} \longrightarrow C^{\infty}\left(\mathbb{R}^{d}\right),
$$

s'écrit :

$$
\begin{aligned}
& \partial C^{\prime}\left(\xi_{0}, \ldots, \xi_{n}\right) \\
& \quad=\sum_{i=0}^{n}(-1)^{i} \mathscr{L}_{\xi_{i}} C^{\prime}\left(\xi_{0}, \ldots \hat{\imath} \ldots, \xi_{n}\right)+\sum_{i<j}(-1)^{i+j} C^{\prime}\left(\left[\xi_{i}, \xi_{j}\right], \xi_{0}, \ldots \hat{\imath} \ldots \hat{\jmath} \ldots, \xi_{n}\right) .
\end{aligned}
$$


On retrouve l'opérateur de cohomologie de Chevalley de l'algèbre de Lie des champs de vecteurs sur le module des 0 -formes défini par la dérivée de Lie $\mathscr{L}$ utilisé en particulier dans [De Wilde et Lecomte 1983].

Soit $C^{\prime}$ une $n$-cochaîne. Posons $C=\tau C^{\prime}$ et définissons $\partial C$ par

$$
\partial C=\tau \partial^{\prime} C^{\prime}
$$

Lemme 2.5 (Cohomologie symétrisée). Une application n-linéaire | |-symétrique $C$ est une $n$-cochaîne. Son cobord $\partial C$ est donné par

$(\partial C)\left(\alpha_{0}, \ldots, \alpha_{n}\right)=$

$$
\begin{aligned}
\sum_{i=0}^{n} \varepsilon_{|\alpha|}(i, 0 \ldots \hat{\imath} \ldots n)(-1)^{|C|\left(\left|\alpha_{i}\right|-1\right)} Q\left(\alpha_{i}, C\left(\alpha_{0}, \ldots \widehat{\alpha}_{i} \ldots, \alpha_{n}\right)\right) \\
\quad-\frac{1}{2} \sum_{i \neq j} \varepsilon_{|\alpha|}(i, j, 0, \ldots \hat{\imath} \ldots \hat{\jmath} \ldots n) C\left(Q\left(\alpha_{i}, \alpha_{j}\right), \alpha_{0}, \ldots \widehat{\alpha}_{i} \ldots \widehat{\alpha}_{j} \ldots, \alpha_{n}\right)
\end{aligned}
$$

ou bien par

$$
\begin{aligned}
& (\partial C)\left(\alpha_{0}, \ldots, \alpha_{n}\right) \\
& \begin{aligned}
=\sum_{i=0}^{n}\left(\varepsilon_{|\alpha|}(i, 0 \ldots \hat{\imath} \ldots n)(-1)^{|C|\left(\left|\alpha_{i}\right|-1\right)} \nabla_{\alpha_{i}} C\left(\alpha_{0}, \ldots \widehat{\alpha}_{i} \ldots, \alpha_{n}\right)\right. \\
\\
\left.\quad+(-1)^{|C|} \varepsilon_{|\alpha|}(0 \ldots \hat{\imath} \ldots n, i) \nabla_{C\left(\alpha_{0}, \ldots \widehat{\alpha}_{i} \ldots, \alpha_{n}\right)} \alpha_{i}\right) \\
\quad-\sum_{i \neq j} \varepsilon_{|\alpha|}(i, j, 0, \ldots \hat{\imath} \ldots \hat{\jmath} \ldots n) C\left(\nabla_{\alpha_{i}} \alpha_{j}, \alpha_{0}, \ldots \widehat{\alpha}_{i} \ldots \widehat{\alpha}_{j} \ldots, \alpha_{n}\right) .
\end{aligned}
\end{aligned}
$$

\section{Formalités et cohomologies}

Cette section consiste en des rappels sur la notion de formalité et sur l'intérêt de la cohomologie de Chevalley sur certains espaces de cochaînes dans leur construction.

Considèrons l'espace $D_{\text {poly }}\left(\mathbb{R}^{d}\right)$ des opérateurs multidifférentiels sur $\mathbb{R}^{d}$ gradué par $\operatorname{deg}(D)=m-1$ si $D$ est un opérateur $m$-différentiel. Sur $D_{\text {poly }}\left(\mathbb{R}^{d}\right)$, on a une multiplication naturelle $\times$ :

$$
\left(D_{1} \times D_{2}\right)\left(f_{1}, \ldots, f_{m_{1}+m_{2}}\right)=D_{1}\left(f_{1}, \ldots, f_{m_{1}}\right) D_{2}\left(f_{m_{1}+1}, \ldots, f_{m_{1}+m_{2}}\right)
$$

et une composition o qui associe à un opérateur différentiel $D_{1}$ et un opérateur $m_{2}$-différentiel $D_{2}$ l'opérateur $m_{2}$-différentiel :

$$
\left(D_{1} \circ D_{2}\right)\left(f_{1}, \ldots, f_{m_{2}}\right)=D_{1}\left(D_{2}\left(f_{1}, \ldots, f_{m_{2}}\right)\right) .
$$

On prolonge à $D_{\text {poly }}\left(\mathbb{R}^{d}\right)$ l'opérateur o en lui imposant les mêmes propriétés que $\nabla$. Pour un opérateur $D_{1} m_{1}$-différentiel et un opérateur $D_{2} m_{2}$-différentiel o 
s'écrit :

$$
\begin{aligned}
& \left(D_{1} \circ D_{2}\right)\left(f_{1}, \ldots, f_{m_{1}+m_{2}-1}\right) \\
& =(-1)^{\left(m_{1}+1\right) m_{2}} \sum_{j=1}^{m_{1}}(-1)^{(j-1)\left(m_{2}-1\right)} D_{1}\left(f_{1}, \ldots, f_{j-1}, D_{2}\left(f_{j}, \ldots, f_{j+m_{2}-1}\right),\right. \\
& \left.f_{j+m_{2}}, \ldots, f_{m_{1}+m_{2}-1}\right) .
\end{aligned}
$$

Le crochet associé à o, appelé "crochet de Gerstenhaber", se calcule comme pour le crochet de Schouten en fonction de $\nabla$. Il s'agit de :

$$
\left[D_{1}, D_{2}\right]_{G}=(-1)^{\operatorname{deg}\left(D_{1}\right)} D_{1} \circ D_{2}-(-1)^{\left(\operatorname{deg}\left(D_{1}\right)+1\right) \operatorname{deg}\left(D_{2}\right)} D_{2} \circ D_{1} .
$$

Ce crochet fait de $D_{\text {poly }}\left(\mathbb{R}^{d}\right)$ une algèbre de Lie graduée. Cette algèbre de Lie a une différentielle donnée par l'opérateur de cobord $d_{H}$ de Hochschild. Plus précisément, on a :

$$
\begin{aligned}
d_{H} D & \left(f_{1}, \ldots, f_{m+1}\right) \\
& =f_{1} D\left(f_{2}, \ldots, f_{m+1}\right)+\sum_{j=1}^{m}(-1)^{j} D\left(f_{1}, \ldots, f_{j} f_{j+1}, \ldots, f_{m+1}\right) \\
& =-[D, \mu]_{G}\left(f_{1}, \ldots, f_{m+1}\right)
\end{aligned}
$$

si $\mu\left(f_{1}, f_{2}\right)=f_{1} f_{2}$ est la multiplication usuelle des fonctions.

Cependant, comme dans le paragraphe 2 , nous prenons ici la graduation $|D|=$ $m$ si $D$ est un opérateur $m$-différentiel. On retrouve l'opérateur symétrique $Q^{\prime}=$ $\tau[,]_{G}$ de [Kontsevich 2003] et [Arnal et al. 2002] sur $D_{\text {poly }}\left(\mathbb{R}^{d}\right)$ :

$$
Q_{1}^{\prime}(D)=-d_{H} D \quad \text { et } \quad Q_{2}^{\prime}\left(D_{1}, D_{2}\right)=D_{1} \circ D_{2}+(-1)^{\left|D_{1}\right|\left|D_{2}\right|} D_{2} \circ D_{1} .
$$

Une formalité sur $\mathbb{R}^{d}$ est une application non linéaire formelle $\mathscr{F}$ entre deux variétés formelles, dont la première est l'espace $T_{\text {poly }}\left(\mathbb{R}^{d}\right)$ gradué par $\operatorname{deg}(\alpha)=k-2$ si $\alpha$ est un $k$-tenseur. Cet espace est muni du champ de vecteurs $Q$ défini dans le paragraphe 2 , de degré 1 et qui vérifie l'équation maîtresse $[Q, Q]=0$ (voir [Kontsevich 2003]). La seconde variété formelle est l'espace $D_{\text {poly }}\left(\mathbb{R}^{d}\right)$ des opérateurs multidifférentiels sur $\mathbb{R}^{d}$ gradué par $\operatorname{deg}(D)=m-2$ si $D$ est un opérateur $m$-différentiel et muni du champ de vecteurs :

$$
Q^{\prime}(D)=Q_{1}^{\prime}(D)+\frac{1}{2} Q_{2}^{\prime}(D, D) .
$$

$Q^{\prime}$ est aussi de degré 1 et vérifie $\left[Q^{\prime}, Q^{\prime}\right]=0$ (voir [Kontsevich 2003]). On dit que $\mathscr{F}_{F}$ est une formalité si elle est de degré 0 et si les deux champs de vecteurs $Q$ et $Q^{\prime}$ sont $\mathscr{F}$-reliés. Ceci se traduit par une suite d'équations sur les termes du développement de Taylor formel $\mathscr{F}_{F}=\sum_{n \geq 1} \mathscr{F}_{n}$, à savoir avec les notations de 
[Arnal et al. 2002] :

$$
\begin{aligned}
& Q_{1}^{\prime}\left(\mathscr{F}_{n}\left(\alpha_{1}, \ldots, \alpha_{n}\right)\right)+\frac{1}{2} \sum_{\substack{I \sqcup J=\{1, \ldots, n\} \\
|I|,|J| \geq 1}} \varepsilon_{|\alpha|}(I, J) Q_{2}^{\prime}\left(\mathscr{F}_{|I|}\left(\alpha_{I}\right), \mathscr{F}_{|J|}\left(\alpha_{J}\right)\right) \\
& \quad=\frac{1}{2} \sum_{i \neq j} \varepsilon_{|\alpha|}(i, j, 1, \ldots \hat{\imath} \ldots \hat{\jmath} \ldots, n) \mathscr{F}_{n-1}\left(Q\left(\alpha_{i}, \alpha_{j}\right), \alpha_{1}, \ldots \widehat{\alpha}_{i} \ldots \widehat{\alpha}_{j} \ldots, \alpha_{n}\right) .
\end{aligned}
$$

On impose aussi

$$
\mathscr{F}_{1}(\alpha)\left(f_{1}, \ldots, f_{m}\right)=\left\langle\alpha, d f_{1} \wedge \cdots \wedge d f_{m}\right\rangle:=\sum_{1 \leq i_{1}, \ldots, i_{m} \leq d} \alpha^{i_{1} \ldots i_{m}} \partial_{i_{1}}\left(f_{1}\right) \ldots \partial_{i_{m}}\left(f_{m}\right) .
$$

Supposons que l'on connaisse $\mathscr{F}_{1}, \ldots, \mathscr{F}_{n-1}$. L'équation de formalité à l'ordre $n$ s'écrit :

$$
d_{H}\left(\mathscr{F}_{n}\left(\alpha_{1}, \ldots, \alpha_{n}\right)\right)=E_{n}\left(\alpha_{1}, \ldots, \alpha_{n}\right) .
$$

Le second membre $E_{n}\left(\alpha_{1}, \ldots, \alpha_{n}\right)$ est par construction un cocycle de Hochschild.

Mais la cohomologie de Hochschild différentielle de $C^{\infty}\left(\mathbb{R}^{d}\right)$ est bien connue : voir [Vey 1975; Berger 1982] par exemple. Si a est l'opérateur d'antisymétrisation

$$
\mathfrak{a}(D)\left(f_{1}, \ldots, f_{m}\right)=\frac{1}{m !} \sum_{\sigma \in S_{m}} \varepsilon(\sigma) D\left(f_{\sigma(1)}, \ldots, f_{\sigma(m)}\right),
$$

alors $D$ est un cocycle de Hochschild si et seulement si $\mathfrak{a}(D)$ est un opérateur multidifférentiel d'ordre $1, \ldots, 1$, c'est à dire si et seulement s'il existe un unique tenseur $\alpha$ dans $T_{\text {poly }}\left(\mathbb{R}^{d}\right)$ tel que :

$$
\mathfrak{a}(D)=\mathscr{F}_{1}(\alpha) .
$$

Enfin, l'application $D \mapsto \alpha$ est un isomorphisme entre la cohomologie de Hochschild et $T_{\text {poly }}\left(\mathbb{R}^{d}\right)$. C'est à dire pour tout cocycle $D, \mathfrak{a}(D)$ est dans la même classe de cohomologie que $D$ et $D$ est un cobord si et seulement si $\mathfrak{a}(D)=0$.

En antisymétrisant l'équation de formalité à l'ordre $n$, on construit donc une cochaîne $R_{n}$ de $T_{\text {poly }}\left(\mathbb{R}^{d}\right)$ telle que :

$$
\mathscr{F}_{1}\left(R_{n}\right)=\mathfrak{a}\left(E_{n}\right) .
$$

L'équation de formalité à l'ordre $n$ a une solution si et seulement si $R_{n}=0$.

L'équation de formalité étant vérifiée à l'ordre $n-1$, on a :

$$
d_{H} \circ \mathscr{F}_{n-1}=E_{n-1} \text { et donc } \mathfrak{a}\left(E_{n-1}\right)=0 .
$$

Si $A_{n-1}$ est une cochaîne de $T_{\text {poly }}\left(\mathbb{R}^{d}\right)$, l'opérateur $\mathscr{F}_{n-1}+\mathscr{F}_{1} \circ A_{n-1}$ satisfait l'équation à l'ordre $n-1$. De plus, à l'ordre $n$ nos relations deviennent :

$$
d_{H} \circ \mathscr{F}_{n}=E_{n}^{\prime}, \quad \mathfrak{a}\left(E_{n}^{\prime}\right)=\mathscr{F}_{1}\left(R_{n}^{\prime}\right), \quad R_{n}^{\prime}=\partial A_{n-1}+R_{n} .
$$

L'équation est maintenant $R_{n}^{\prime}=0$. 
Proposition 3.1 (Formalité et cohomologie de Chevalley [Kontsevich 2003; Arnal et al. 2005]). L'obstruction au prolongement de la construction des $\mathscr{F}_{n}$ est localisée dans la cohomologie de Chevalley de $T_{\mathrm{poly}}\left(\mathbb{R}^{d}\right)$.

Dans le paragraphe suivant, nous allons construire des applications de $T_{\text {poly }}\left(\mathbb{R}^{d}\right)$ dans $D_{\text {poly }}\left(\mathbb{R}^{d}\right)$ universelles à l'aide des graphes de Kontsevich. Cette construction ne dépend pas de la dimension $d$.

On va donc ici étendre les constructions précédentes à $\mathbb{R}^{\infty}$, la limite inductive des $\mathbb{R}^{d}$. Plus précisément, $\mathbb{R}^{\infty}$ est l'espace vectoriel de base $\left(e_{1}, \ldots, e_{d}, \ldots\right)$, l'espace $\mathbb{R}^{d}$ est identifié au sous-espace engendré par $\left(e_{1}, \ldots, e_{d}\right)$ de $\mathbb{R}^{\infty}$, et les injections canoniques définissent un système inductif tel que

$$
\mathbb{R}^{\infty}=\lim ^{\longrightarrow} \mathbb{R}^{d}
$$

Notons $\left(x_{1}, \ldots, x_{d}\right)$ les coordonnées de $x$ dans $\mathbb{R}^{d}$ sur la base $\left(e_{1}, \ldots, e_{d}\right)$. L'espace $T_{\text {poly }}^{k}\left(\mathbb{R}^{d}\right)$ est l'ensemble des tenseurs

$$
\alpha=\sum_{1 \leq i_{1}, \ldots, i_{k} \leq d} \alpha^{i_{1}, \ldots, i_{k}}\left(x_{1}, \ldots, x_{d}\right) \partial_{i_{1}} \wedge \cdots \wedge \partial_{i_{k}} .
$$

On identifie cet espace à un sous-espace de $T_{\text {poly }}^{k}\left(\mathbb{R}^{d+1}\right)$ de façon canonique. On obtient un nouveau système inductif et on pose

$$
T_{\text {poly }}^{k}\left(\mathbb{R}^{\infty}\right)=\bigcup_{d=1}^{\infty} T_{\text {poly }}^{k}\left(\mathbb{R}^{d}\right) \quad \text { et } \quad T_{\text {poly }}\left(\mathbb{R}^{\infty}\right)=\bigoplus_{k=0}^{\infty} T_{\text {poly }}^{k}\left(\mathbb{R}^{\infty}\right) .
$$

De même, $D_{\text {poly }}\left(\mathbb{R}^{\infty}\right)$ est la somme directe des espaces

$$
D_{\text {poly }}^{m}\left(\mathbb{R}^{\infty}\right)=\bigcup_{d=1}^{\infty} D_{\text {poly }}^{m}\left(\mathbb{R}^{d}\right),
$$

union des opérateurs $m$-différentiels sur $\mathbb{R}^{d}$. Il est facile de voir que :

Proposition 3.2 (Les algèbres $T_{\text {poly }}\left(\mathbb{R}^{\infty}\right)$ et $D_{\text {poly }}\left(\mathbb{R}^{\infty}\right)$ ). L'espace $T_{\text {poly }}\left(\mathbb{R}^{d}\right)$ (resp. $D_{\text {poly }}\left(\mathbb{R}^{d}\right)$ ) est un sous-espace vectoriel de $T_{\text {poly }}\left(\mathbb{R}^{\infty}\right)$ (resp. de $D_{\text {poly }}\left(\mathbb{R}^{\infty}\right)$ ). On a:

$$
T_{\text {poly }}\left(\mathbb{R}^{\infty}\right)=\underline{\lim } T_{\text {poly }}\left(\mathbb{R}^{d}\right) \quad \text { et } \quad D_{\text {poly }}\left(\mathbb{R}^{\infty}\right)=\underline{\lim } D_{\text {poly }}\left(\mathbb{R}^{d}\right) .
$$

Les opérations $\wedge$ et $[],\left(\right.$ resp. $\times$ et $\left.[,]_{G}\right)$ définissent des systèmes inductifs d'applications et se prolongent de façon unique à $T_{\text {poly }}\left(\mathbb{R}^{\infty}\right)\left(\right.$ resp. à $\left.D_{\text {poly }}\left(\mathbb{R}^{\infty}\right)\right)$.

L'espace des fonctions sur $\mathbb{R}^{\infty}$ est l'espace $T_{\text {poly }}^{0}\left(\mathbb{R}^{\infty}\right)$, c'est à dire l'espace des fonctions $f$ de classe $C^{\infty}$ ne dépendant que d'un nombre fini de variables : $f(x)=$ $f\left(x_{1}, \ldots, x_{D}\right)$. 
Définition 3.3 (Cochaînes inductives). Une cochaîne inductive à valeurs dans l'espace $T_{\text {poly }}\left(\mathbb{R}^{\infty}\right)$ (resp. $\left.D_{\text {poly }}\left(\mathbb{R}^{\infty}\right)\right)$ est une suite d'applications $C_{d}, n$-linéaires, | |symétriques qui forme un ensemble inductif, c'est à dire telle qu'il existe $D$ tel que, pour tout $d \geq D$,

$$
C_{d}\left(\left(T_{\text {poly }}\left(\mathbb{R}^{d}\right)\right)^{n}\right) \subset T_{\text {poly }}\left(\mathbb{R}^{d}\right) \quad\left(\text { resp. } D_{\text {poly }}\left(\mathbb{R}^{d}\right)\right) .
$$

Un premier exemple de telle cochaîne est donné par les cochaînes différentielles. Soit $D$ un entier, $a=\left(a^{1}, \ldots, a^{D}\right) \in \mathbb{N}^{D}$ un multi-indice. On définit l'opérateur

$$
\left(\partial_{x}\right)^{a}=\partial_{1}^{a^{1}} \circ \cdots \circ \partial_{D}^{a^{D}} .
$$

Une cochaîne $C$ est dite différentielle s'il existe un nombre fini de tenseurs

$$
A_{a_{1}, \ldots, a_{n}, i_{1}, \ldots, i_{k_{1}}, \ldots, j_{1}, \ldots, j_{k_{n}}}\left(x_{1}, \ldots, x_{D}\right)
$$

dans $T_{\text {poly }}\left(\mathbb{R}^{D}\right)$ (resp. d'opérateurs multidifférentiels dans $D_{\text {poly }}\left(\mathbb{R}^{D}\right)$ ) tels que :

$$
C\left(\alpha_{1}, \ldots, \alpha_{n}\right)=\sum_{\text {finie }} A_{a_{1}, \ldots, a_{n}, i_{1}, \ldots, i_{k_{1}}, \ldots, j_{1}, \ldots, j_{k_{n}}}(x)\left(\partial_{x}\right)^{a_{1}} \alpha_{1}^{i_{1} \ldots i_{k_{1}}} \ldots\left(\partial_{x}\right)^{a_{n}} \alpha_{n}^{j_{1} \ldots j_{k_{n}}} .
$$

Une cochaîne différentielle est inductive par construction. Les cochaînes étudiées par [De Wilde et Lecomte 1983] sont des cochaînes différentielles. Une autre classe de cochaînes inductives est donnée par les constructions de Kontsevich que nous rappelons dans le paragaphe suivant.

\section{Graphes aériens et cochaines}

Dans cet article, on considère des graphes de Kontsevich, c'est à dire des graphes $\Gamma$ ayant des sommets aériens numérotés $1, \ldots, n$, que l'on peut voir comme des points du demi espace de Poincaré $\{\operatorname{Im} z>0\}$, et des sommets terrestres numérotés $\overline{1}, \ldots, \bar{m}$, que l'on peut voir comme des points rangés sur l'axe réel. De chaque sommet aérien $i$ partent $k_{i} \geq 0$ arêtes du graphe. Ces arêtes sont des flèches d'extrémité soit un sommet aérien soit un sommet terrestre. (On s'autorise des "petites boucles", c'est à dire des arêtes qui reviennent au sommet de départ.) Il n'y a pas d'arête partant d'un sommet terrestre, appelé pied du graphe, et il y a exactement une arête y arrivant; cette arête est une jambe du graphe. Il n'y a pas d'arête multiple (mais on peut avoir les arêtes $i j$ et $j i$ si $i \neq j$ ).

Fixons un ordre 0 sur les arêtes (c'est à dire une numérotation des flèches de $\Gamma$ ) qui soit compatible avec la numérotation des sommets aériens c'est à dire tel que les $k_{1}$ premières flèches partent du sommet 1 , les $k_{2}$ suivantes du sommet 2 , etc. On définit alors une application $B_{\Gamma, \odot} n$-linéaire de $T_{\text {poly }}\left(\mathbb{R}^{d}\right)$ dans lui même de la façon suivante : 
$B_{\Gamma, \mathcal{O}}\left(\alpha_{1}, \ldots, \alpha_{n}\right)$ est nul sauf si $\alpha_{1}$ est un $k_{1}$-tenseur, $\alpha_{2}$ un $k_{2}$-tenseur, $\ldots, \alpha_{n}$ un $k_{n}$-tenseur.

Dans ce dernier cas, on appelle Deb $(i)$ l'ensemble ordonné des flèches issues de $i$, elles portent les numéros $a_{i}+1:=\left(\sum_{j<i} k_{j}\right)+1, a_{i}+2, \ldots, a_{i}+k_{i}=\sum_{j \leq i} k_{j}$. On appelle aussi Fin $(i)$ et Fin $(\bar{l})$ l'ensemble des flèches arrivant sur le sommet $i$, respectivement $\bar{l}$, elles portent les numéros $s_{1}^{i}, \ldots, s_{r_{i}}^{i}$, respectivement $s^{\bar{l}}$.

On pose $|k|=k_{1}+\cdots+k_{n}$ et on considère tous les multi-indices $\left(t_{1}, \ldots, t_{|k|}\right)$ variant entre 1 et $d$, la dimension de l'espace $\mathbb{R}^{d}$. Chaque $t$ est donc une application de l'ensemble des flèches dans $\{1, \ldots, d\}$. On note $t(\operatorname{Deb}(i))$ la suite ordonnée des indices $\left(t_{\left(a_{i}+1\right)}, \ldots, t_{\left(a_{i}+k_{i}\right)}\right)$, on note aussi $t(\operatorname{Fin}(i))$ la suite $\left(t_{s_{1}^{i}}, \ldots, t_{s_{r_{i}^{i}}^{i}}\right)$ et $t(\operatorname{Fin}(\bar{l}))=t_{s^{\bar{l}}}$.

On notera les composantes des tenseurs $\alpha_{i}$ :

$$
\alpha_{i}^{t(\operatorname{Deb}(i))}:=\alpha_{i}^{t_{\left(a_{i}+1\right) \ldots t_{\left(a_{i}+k_{i}\right)}}} .
$$

De même, on définit des opérateurs de dérivations :

$$
\partial_{t(\operatorname{Fin}(i))}=\frac{\partial^{r_{i}}}{\partial x^{t_{s_{1}^{i}}^{i}} \ldots \partial x^{t_{s_{i}}}}:=\partial_{t_{s_{1}} \ldots t_{s_{r_{i}}}} \quad \text { et } \quad \partial_{t(\operatorname{Fin}(\bar{\imath}))}:=\partial_{t_{s^{\bar{i}}}} \text {. }
$$

Alors on définit $B_{\Gamma, \mathcal{O}}\left(\alpha_{1}, \ldots, \alpha_{n}\right)$ par :

$$
B_{\Gamma, \mathcal{O}}\left(\alpha_{1}, \ldots, \alpha_{n}\right)=\sum_{1 \leq t_{1}, \ldots, t_{|k|} \leq d} \prod_{i=1}^{n} \partial_{t(\operatorname{Fin}(i))} \alpha_{i}^{t(\operatorname{Deb}(i))} \partial_{t(\operatorname{Fin}(\overline{1}))} \wedge \cdots \wedge \partial_{t(\operatorname{Fin}(\bar{m}))}
$$

Cet opérateur est défini pour tout $d$, il forme un système inductif d'applications, on obtient ainsi une application de $\left(T_{\text {poly }}\left(\mathbb{R}^{\infty}\right)\right)^{n}$ dans $T_{\text {poly }}\left(\mathbb{R}^{\infty}\right)$, toujours notée $B_{\Gamma, \mathcal{O}}$. Si $a_{\Gamma, \mathcal{O}}\left(x_{1}, \ldots, x_{D}\right)$ est une fonction sur $\mathbb{R}^{\infty}$, le système d'applications $a_{\Gamma, \mathcal{O}}(x) B_{\Gamma, \mathcal{O}}$ de $\left(T_{\text {poly }}\left(\mathbb{R}^{d}\right)\right)^{n}$ dans $T_{\text {poly }}\left(\mathbb{R}^{d}\right)(d \geq D)$ est aussi inductif et définit une application, toujours notée $a_{\Gamma, \mathcal{O}}(x) B_{\Gamma, \mathcal{O}}$ de $\left(T_{\text {poly }}\left(\mathbb{R}^{\infty}\right)\right)^{n}$ dans $T_{\text {poly }}\left(\mathbb{R}^{\infty}\right)$.

Remarque 4.1. La définition de l'opérateur $B_{\Gamma, \bigcirc}$ dépend du choix de l'ordre compatible $\mathcal{O}$. Changer cet ordre revient à multiplier $B_{\Gamma, \mathcal{O}}$ par le signe noté $\varepsilon\left(\mathcal{O}, \mathcal{O}^{\prime}\right)$ dans [Arnal et al. 2005] de la permutation de l'ensemble des arêtes faisant passer de 0 à $\mathrm{O}^{\prime}$.

On étend la définition de l'opérateur $B_{\Gamma, \odot}$ aux ordres $O^{\prime}$ non compatibles en posant

$$
B_{\Gamma, \mathcal{O}^{\prime}}=\varepsilon\left(\mathcal{O}, \mathrm{O}^{\prime}\right) B_{\Gamma, \mathcal{O}}
$$

Si on se restreint aux graphes vectoriels, c'est à dire aux graphes tels que $\operatorname{Deb}(i)$ a au plus un élément, il y a un seul ordre compatible. On numérotera alors les flèches par le numéro $i$ de leur origine.

Un graphe qui n'a aucun pied et aucune jambe est appelé graphe aérien. Si $\Delta$ est un tel graphe aérien, on peut le "compléter" en lui ajoutant des pieds et 
des jambes. On considérera donc tous les graphes $\Gamma$ tels que, lorsque l'on retire les pieds et les jambes de $\Gamma$, on retrouve $\Delta$. Si $(\Delta, \mathcal{O})$ est un graphe orienté, on considérera tous les graphes orientés $\left(\Gamma, O_{\Gamma}\right)$ tels que l'ordre induit par $O_{\Gamma}$ sur l'ensemble des arêtes qui ne sont pas des jambes soit exatement 0 . On notera cette propriété $\left(\Gamma, O_{\Gamma}\right) \supset(\Delta, \mathcal{O})$.

Plus précisément, on reprendra la définition de [Arnal et al. 2005]. Partant de $(\Delta, 0)$ où $O$ est compatible et de $m$ pieds numérotés $\overline{1}, \ldots, \bar{m}$, on peut construire un graphe $\Gamma$ en ajoutant $m$ jambes à $\Delta$ (une pour chaque pied). On peut définir un ordre $O_{0}$ sur les arêtes de $\Gamma$ en rangeant les jambes après les arêtes aériennes, dans l'ordre des pieds. Si $k_{1}, \ldots, k_{n}$ sont les nombres d'arêtes de $\Gamma$ issues des sommets $1, \ldots, n$ et $\ell_{1}, \ldots, \ell_{n}$ le nombre d'arêtes de $\Delta$ issues des sommets $1, \ldots, n$, il y a

$$
\frac{k !}{\ell !}=\frac{k_{1} ! \ldots k_{n} !}{\ell_{1} ! \ldots \ell_{n} !}
$$

ordres compatibles possibles $\mathscr{O}_{\Gamma}$ tels que $\left(\Gamma, \mathscr{O}_{\Gamma}\right) \supset(\Delta, \mathcal{O})$.

On posera donc :

$$
C_{\Delta, \mathcal{O}}=\sum_{m=0}^{\infty} \frac{1}{m !} \sum_{\substack{\left(\Gamma, \mathcal{C}_{\Gamma}\right) \supset(\Delta, \mathcal{O}) \\ \#\{\text { pieds de } \Gamma\}=m}} \frac{\ell !}{k !} \varepsilon\left(\mathscr{O}_{\Gamma}, \mathscr{O}_{0}\right) B_{\Gamma, \mathcal{O}_{\Gamma}} .
$$

Cette série définit encore une application de $\left(T_{\text {poly }}\left(\mathbb{R}^{d}\right)\right)^{n}$ dans $T_{\text {poly }}\left(\mathbb{R}^{d}\right)($ dans ce cas la somme est finie, $m \leq d)$ ou une application de $\left(T_{\text {poly }}\left(\mathbb{R}^{\infty}\right)\right)^{n}$ dans $T_{\text {poly }}\left(\mathbb{R}^{\infty}\right)$ (dans ce cas la somme est infinie).

Les opérateurs $C_{\Delta, \mathcal{O}}$ apparaissent dans la construction de Kontsevich d'une formalité $\mathscr{F}=\sum_{n} \mathscr{F}_{n}$ explicite et universelle sur $\mathbb{R}^{d}$, puisqu'il est montré dans [Arnal et al. 2005] que

$$
\mathfrak{a} \circ \mathscr{F}_{n}\left(\alpha_{1}, \ldots, \alpha_{n}\right)=\sum_{\Delta, \mathcal{O}} w_{\Delta, \mathcal{O}} C_{\Delta, \mathcal{O}}\left(\alpha_{1}, \ldots, \alpha_{n}\right)
$$

les nombres $w_{\Delta, \mathcal{O}}$ sont des constantes définies par Kontsevich et appelées poids du graphe orienté $\Delta, \mathcal{O}$.

Lorsque l'on se restreint aux graphes vectoriels, la série $C_{\Delta, \mathcal{O}}$ est une somme finie. Elle ne contient que des graphes $\Gamma$ ayant $m$ pieds avec :

$$
0 \leq m \leq n-|\ell| \text {. }
$$

De plus, il n'y a qu'un ordre compatible $\mathscr{O}_{\Delta}$ sur les arêtes de $\Delta$, on notera donc simplement $C_{\Delta}$ l'opérateur $C_{\Delta, \mathscr{O}_{\Delta}}$.

Si $\sigma$ est une permutation de $n$ éléments, $\sigma$ agit sur $\Delta$ en permutant ses sommets. Dans [Arnal et al. 2005], il est montré que :

$$
C_{\sigma(\Delta)}\left(\alpha_{\sigma(1)}, \ldots, \alpha_{\sigma(n)}\right)=C_{\Delta}\left(\alpha_{1}, \ldots, \alpha_{n}\right) .
$$


Pour construire des cochaînes, on considère donc des combinaisons symétriques de graphes vectoriels $\Delta$.

Définition 4.2 (Cochaînes et graphes vectoriels symétriques). Une combinaison linéaire sur $C^{\infty}\left(\mathbb{R}^{d}\right)$ ou $T_{\text {poly }}^{0}\left(\mathbb{R}^{\infty}\right)$ de graphes vectoriels aériens $\Delta$ ayant $n$ sommets,

$$
\delta=\sum_{\Delta} a_{\Delta}(x) \Delta
$$

est dite symétrique si elle vérifie, pour tout $\Delta$ et tout $\sigma$,

$$
a_{\sigma(\Delta)}(x)=\varepsilon_{|\operatorname{Deb}|}(\sigma) a_{\Delta}(x)
$$

où $|\operatorname{Deb}|=(|\operatorname{Deb}(1)|, \ldots,|\operatorname{Deb}(n)|)$; on rappelle que $|\operatorname{Deb}(i)|$ est le nombre de flèches issues de $i$.

Une $n$-cochaîne $C$ est dite associée à un graphe symétrique s'il existe une combinaison linéaire symétrique $\delta$ telle que

$$
C=C_{\delta}=\sum_{\Delta} a_{\Delta}(x) C_{\Delta} .
$$

Par exemple, la roue simple $\Delta$ de longueur 3 est un graphe aérien à 3 sommets numérotés $1,2,3$ et ayant pour arêtes $\{12,23,31\}$. C'est un graphe vectoriel. $\mathrm{La}$ symétrisation $\delta=R_{3}$ de $\Delta$ est :

$$
\delta=\sum_{\sigma \in S_{3}} \varepsilon(\sigma) \sigma(\Delta)=3 \times\{12,23,31\}-3 \times\{13,32,21\} .
$$

Cette symétrisation définit une application $C_{\delta}$ qui envoie trois champs de vecteurs $\alpha_{1}, \alpha_{2}, \alpha_{3}$ sur la fonction

$$
C_{\delta}\left(\alpha_{1}, \alpha_{2}, \alpha_{3}\right)=3 \partial_{i_{3}} \alpha_{1}^{i_{1}} \partial_{i_{1}} \alpha_{2}^{i_{2}} \partial_{i_{2}} \alpha_{3}^{i_{3}}-3 \partial_{i_{2}} \alpha_{1}^{i_{1}} \partial_{i_{1}} \alpha_{3}^{i_{3}} \partial_{i_{3}} \alpha_{2}^{i_{2}} .
$$

Proposition 4.3 ( $C$ est injective). Soit $V_{n}$ l'espace des combinaisons linéaires $\delta=$ $\sum a_{\Delta}(x) \Delta$ de graphes vectoriels aériens ayant $n$ sommets. Alors l'application:

$$
C: V_{n} \longrightarrow \mathscr{L}^{n}\left(T_{\text {poly }}\left(\mathbb{R}^{\infty}\right), T_{\text {poly }}\left(\mathbb{R}^{\infty}\right)\right)
$$

est injective.

Démonstration. Supposons que $\delta$ est un élément non nul du noyau de $C$. Pour chaque $\Delta$, on note $|\Delta|$ le nombre de ses flèches, on écrit donc :

$$
\delta=\sum_{\ell=0}^{n} \sum_{|\Delta|=\ell} a_{\Delta}(x) \Delta .
$$

Soit $D$ tel que $a_{\Delta}(x)=a_{\Delta}\left(x_{1}, \ldots, x_{D}\right)$ pour tout $\Delta$. 
On ordonne les graphes suivant l'ordre lexicographique, c'est à dire qu'une flèche $i j$ est plus petite qu'une flèche $i^{\prime} j^{\prime}$ si $i<i^{\prime}$, et aussi que le graphe $\Delta=$ $\left\{i_{1} j_{1}, \ldots, i_{\ell} j_{\ell}\right\}$ est plus petit que $\Delta^{\prime}=\left\{i_{1}^{\prime} j_{1}^{\prime}, \ldots, i_{\ell^{\prime}}^{\prime} j_{\ell^{\prime}}^{\prime}\right\}\left(\Delta<\Delta^{\prime}\right)$ si

$$
|\Delta|<\left|\Delta^{\prime}\right| \quad \text { ou }\left\{\begin{array} { c } 
{ | \Delta | = | \Delta ^ { \prime } | } \\
{ \text { et } } \\
{ i _ { 1 } j _ { 1 } < i _ { 1 } ^ { \prime } j _ { 1 } ^ { \prime } }
\end{array} \quad \text { ou } \left\{\begin{array}{c}
|\Delta|=\left|\Delta^{\prime}\right| \\
\text { et } \\
i_{1} j_{1}=i_{1}^{\prime} j_{1}^{\prime} \quad \ldots \\
\text { et }
\end{array}\right.\right.
$$

Soit $\Delta_{0}$ le plus petit graphe $\Delta$ tel que $a_{\Delta}(x) \neq 0$. Notons $a_{1}, \ldots, a_{\ell}$ les sommets $s$ de $\Delta_{0}$ tels que $\operatorname{Deb}_{\Delta_{0}}(s)$ n'est pas vide et $b_{1}, \ldots, b_{n-\ell}$ les autres. On définit $t:\left\{a_{1}, \ldots, a_{\ell}\right\} \rightarrow\{D+1, D+\ell\}$, en posant

$$
t\left(a_{k}\right)=D+k
$$

On définit les champs de vecteurs $\alpha_{a_{k}}$ et les fonctions $\alpha_{b_{j}}$ par

$$
\alpha_{a_{k}}=\left(\prod_{r \in \operatorname{Fin}_{\Delta_{0}}\left(a_{k}\right)} x_{t(r)}\right) \partial_{D+k}, \quad \alpha_{b_{j}}=\prod_{r \in \operatorname{Fin}_{\Delta_{0}}\left(b_{j}\right)} x_{t(r)} .
$$

Avec ce choix, on a $C_{\Delta_{0}}\left(\alpha_{1}, \ldots, \alpha_{n}\right)=1$. Soit $\Delta$ tel que $a_{\Delta}(x) \neq 0$, et supposons $C_{\Delta}\left(\alpha_{1}, \ldots, \alpha_{n}\right) \neq 0$. Par construction, $|\Delta|=\ell$ et pour chaque $i$ les flèches de $\operatorname{Fin}_{\Delta}(i)$ doivent nécessairement être les flèches avec numéros dans l'ensemble $\operatorname{Fin}_{\Delta_{0}}(i) ;$ c'est à dire que

$$
\operatorname{Fin}_{\Delta}(i)=\operatorname{Fin}_{\Delta_{0}}(i)
$$

De même la flèche qui part éventuellement de $i$ correspond à la seule composante non nulle de $\alpha_{i}$, alors pour tout $i$,

$$
\operatorname{Deb}_{\Delta}(i)=\operatorname{Deb}_{\Delta_{0}}(i)
$$

On a donc $\Delta=\Delta_{0}$ et $C_{\delta}\left(\alpha_{1}, \ldots, \alpha_{n}\right)=a_{\Delta_{0}}(x) \neq 0$, ce qui est absurde.

Corollaire 4.4 (Les cocycles ont des coefficients constants). Soit $\delta=\sum a_{\Delta}(x) \Delta$ une combinaison linéaire symétrique de graphes vectoriels ayant n sommets. Si $C_{\delta}$ est un cocycle, alors les $a_{\Delta}(x)$ sont toutes des constantes.

Démonstration. Elle repose sur la formule de Cartan. Soit $\partial_{k}$ la dérivation par rapport à $x_{k}$, on a :

$$
\mathscr{L}_{\partial_{k}} C_{\delta}=\left(\partial \circ \iota_{\partial_{k}}+\iota_{\partial_{k}} \circ \partial\right) C_{\delta}
$$


En effet, si $C$ est une cochaîne,

$$
\begin{aligned}
& \partial\left(\iota_{\partial_{k}} C\right)\left(\alpha_{1}, \ldots, \alpha_{n}\right) \\
& =\sum_{i=1}^{n}\left(\varepsilon_{|\alpha|}(i, 1 \ldots \hat{\imath} \ldots n)(-1)^{\left|\iota_{\partial_{k}} C\right|\left(\left|\alpha_{i}\right|-1\right)} \nabla_{\alpha_{i}} C\left(\partial_{k}, \alpha_{1}, \ldots \widehat{\alpha}_{i} \ldots, \alpha_{n}\right)\right. \\
& \left.+(-1)^{\left|\iota_{\partial_{k}} C\right|} \varepsilon_{|\alpha|}(1 \ldots \hat{\imath} \ldots n, i) \nabla_{C\left(\partial_{k}, \alpha_{1}, \ldots \widehat{\alpha}_{i} \ldots, \alpha_{n}\right)} \alpha_{i}\right) \\
& -\sum_{i \neq j} \varepsilon_{|\alpha|}(i, j, 1, \ldots \hat{\imath} \ldots \hat{\jmath} \ldots n) C\left(\partial_{k}, \nabla_{\alpha_{i}} \alpha_{j}, \alpha_{1}, \ldots \widehat{\alpha}_{i} \ldots \widehat{\alpha}_{j} \ldots, \alpha_{n}\right) .
\end{aligned}
$$

Remarquons que $\left|\iota_{\partial_{k}} C\right|=|C|+1$ et que $\nabla_{\alpha_{i}} \neq 0$ seulement si $\left|\alpha_{i}\right|=1$. En posant $\alpha_{0}=\partial_{k}$, on obtient d'autre part :

$$
\begin{aligned}
& \iota_{\partial_{k}}(\partial C)\left(\alpha_{1}, \ldots, \alpha_{n}\right) \\
& =\nabla_{\partial_{k}}\left(C\left(\alpha_{1}, \ldots, \alpha_{n}\right)\right) \\
& \quad+\sum_{i=1}^{n}\left(\varepsilon_{|\alpha|}(i, 0,1 \ldots \hat{\imath} \ldots n)(-1)^{|C|\left(\left|\alpha_{i}\right|-1\right)} \nabla_{\alpha_{i}} C\left(\partial_{k}, \alpha_{1}, \ldots \widehat{\alpha}_{i} \ldots, \alpha_{n}\right)\right. \\
& \left.\quad+(-1)^{|C|} \varepsilon_{|\alpha|}(0, \ldots \hat{\imath} \ldots n, i) \nabla_{C\left(\partial_{k}, \alpha_{1}, \ldots \widehat{\alpha}_{i} \ldots, \alpha_{n}\right)} \alpha_{i}\right) \\
& \quad-\sum_{j=1}^{n} \varepsilon_{|\alpha|}(0, j, 1, \ldots \hat{\jmath} \ldots n) C\left(\nabla_{\partial_{k}} \alpha_{j}, \alpha_{1}, \ldots \widehat{\alpha}_{j} \ldots, \alpha_{n}\right) \\
& \quad-\sum_{0<i \neq j} \varepsilon_{|\alpha|}(i, j, 0,1, \ldots \hat{\imath} \ldots \hat{\jmath} \ldots n) C\left(\nabla_{\alpha_{i}} \alpha_{j}, \partial_{k}, \alpha_{1}, \ldots \widehat{\alpha}_{i} \ldots \widehat{\alpha}_{j} \ldots, \alpha_{n}\right) .
\end{aligned}
$$

En remarquant que $\varepsilon_{|\alpha|}(i, 0,1 \ldots \hat{\imath} \ldots n)=-\varepsilon_{|\alpha|}(i, 1 \ldots \hat{\imath} \ldots n)$ lorsque $\nabla_{\alpha_{i}} \neq 0$ (où $\alpha_{i}$ est un champ de vecteurs) et que

$$
\begin{aligned}
\varepsilon_{|\alpha|}(i, j, 0,1, \ldots \hat{\imath} \ldots \hat{\jmath} \ldots n) C\left(\nabla_{\alpha_{i}} \alpha_{j}, \partial_{k}, \alpha_{1}, \ldots \widehat{\alpha}_{i} \ldots \widehat{\alpha}_{j} \ldots, \alpha_{n}\right) \\
\quad=-\varepsilon_{|\alpha|}(i, j, 1, \ldots \hat{\imath} \ldots \hat{\jmath} \ldots n) C\left(\partial_{k}, \nabla_{\alpha_{i}} \alpha_{j}, \alpha_{1}, \ldots \widehat{\alpha}_{i} \ldots \widehat{\alpha}_{j} \ldots, \alpha_{n}\right)
\end{aligned}
$$

puisque $\left|\nabla_{\alpha_{i}} \alpha_{j}\right|=\left|\alpha_{i}\right|+\left|\alpha_{j}\right|-1$, on obtient :

$$
\begin{aligned}
\left(\partial\left(\iota_{\partial_{k}} C\right)+\iota_{\partial_{k}}(\partial C)\right)\left(\alpha_{1}, \ldots\right. & \left., \alpha_{n}\right) \\
& =\nabla_{\partial_{k}}\left(C\left(\alpha_{1}, \ldots, \alpha_{n}\right)\right)-\sum_{j=1}^{n} C\left(\alpha_{1}, \ldots, \nabla_{\partial_{k}} \alpha_{j}, \ldots, \alpha_{n}\right) \\
& =\left(\mathscr{L}_{\partial_{k}} C\right)\left(\alpha_{1}, \ldots, \alpha_{n}\right) .
\end{aligned}
$$

Supposons que $C_{\delta}$ soit un cocycle et appelons $D$ l'indice tel que $a_{\Delta}(x)=$ $a_{\Delta}\left(x_{1}, \ldots, x_{D}\right)$ pour tout $\Delta$. Prenons $k \leq D$ et des tenseurs $\alpha_{i}$ dont les coefficients $\alpha_{i}^{j_{1} \ldots j_{k}}$ sont nuls si l'un des $j_{r} \leq D$ et dans les autres cas ne sont fonctions que des $x_{j}, j>D$. On notera cette propriété $\alpha_{i} \in T_{\text {poly }}\left(\mathbb{R}^{\infty} \backslash \mathbb{R}^{D}\right)$. Dans la preuve précédente, on a montré que si $C_{\delta} \mathrm{s}$ 'annule sur $\left(T_{\text {poly }}\left(\mathbb{R}^{\infty} \backslash \mathbb{R}^{D}\right)\right)^{n}$, alors $\delta$ est nul.

Si $\beta_{1}, \ldots, \beta_{n-1}$ appartiennent à $T_{\text {poly }}\left(\mathbb{R}^{\infty} \backslash \mathbb{R}^{D}\right)$, alors, pour tout $\Delta$,

$$
\left(\iota_{\partial_{k}} C_{\Delta}\right)\left(\beta_{1}, \ldots, \beta_{n-1}\right)=0
$$


puisque la flèche issue du premier sommet de $\Delta$ aboutit sur un tenseur ne dépendant pas de $x_{k}$, la dérivation correspondante s'annule.

Alors :

$\mathscr{L}_{\partial_{k}} C_{\delta}\left(\alpha_{1}, \ldots, \alpha_{n}\right)=C_{\sum \partial_{k}\left(a_{\Delta}\right) \Delta}\left(\alpha_{1}, \ldots, \alpha_{n}\right)=\partial\left(\sum a_{\Delta} \iota_{\partial_{k}} C_{\Delta}\right)\left(\alpha_{1}, \ldots, \alpha_{n}\right)=0$.

Donc $\sum \partial_{k}\left(a_{\Delta}\right) \Delta=0$ et chaque fonction $a_{\Delta}$ est constante.

\section{L'opérateur de cobord sur les graphes vectoriels}

Dans ce paragraphe, nous définissons directement sur les combinaisons linéaires $\delta$ symétriques de graphes aériens vectoriels un opérateur de cobord, toujours noté $\partial$, correspondant à l'opérateur de cobord pour les cochaînes symétriques $C_{\delta}$ définies par $\delta$. Dans [Arnal et al. 2005], il est montré qu'un tel opérateur défini sur les graphes existe : $\partial \delta$ vérifie $C_{\partial \delta}=\partial C_{\delta}$. Dans le cas des graphes vectoriels, son expression peut être simplifiée.

Définition 5.1 (Éclatement propre d'un sommet). Soit $\Delta$ un graphe vectoriel aérien de sommets numérotés $1, \ldots, n$. Fixons $i$ et $j$ tels que $0 \leq i, j \leq n$. On construit une famille de graphes $\Delta_{j i}^{\prime}$ (resp. $\Delta_{i j}^{\prime}$ ) de la façon suivante :

On renumérote les sommets de $\Delta$ en $0, \ldots \hat{\jmath} \ldots, n$ (en gardant leur ordre initial).

$\underline{\text { Si le sommet } i \text { est un point isolé }(\operatorname{Deb}(i)=\operatorname{Fin}(i)=\varnothing)}$

On ajoute le sommet $j$ et la flèche $j i$ (resp. $i j$ ).

$\underline{\text { Si le sommet } i \text { n'est pas un point isolé }}$

On ajoute un sommet $j$ et une flèche $j i$ (resp. $i j$ ),

Si une flèche $i a$ partait de $i$ dans $\Delta$, on la garde dans $\Delta_{j i}^{\prime}$ (resp. on la remplace par $j a$ dans $\Delta_{i j}^{\prime}$ ),

On répartit les flèches du graphe $\Delta$ arrivant sur le sommet $i$ entre les sommets $j$ et $i$ du nouveau graphe $\Delta_{j i}^{\prime}$ (resp. $\Delta_{i j}^{\prime}$ ) proprement, c'est à dire de telle façon que :

$$
\inf (|\operatorname{Deb}(i)|+|\operatorname{Fin}(i)|,|\operatorname{Deb}(j)|+|\operatorname{Fin}(j)|)>1 .
$$

Remarquons que :

- Si $i$ est un sommet isolé, il y a un seul graphe $\Delta_{j i}^{\prime}$.

- Si $i$ est tel que $|\operatorname{Deb}(i)|+|\operatorname{Fin}(i)|=1$ alors il n'y a aucun graphe $\Delta_{j i}^{\prime}$.

- Si $i$ est un sommet tel que $|\operatorname{Deb}(i)|=|\operatorname{Fin}(i)|=1$, il y a un seul $\Delta_{j i}^{\prime}$.

En général si $|\operatorname{Deb}(i)|=1$, il y a $2^{|\operatorname{Fin}(i)|}-1$ graphes $\Delta_{j i}^{\prime} ; \operatorname{si}|\operatorname{Deb}(i)|=0$ et $\mid$ Fin $(i) \mid \geq 1$, il y a $2^{|\operatorname{Fin}(i)|}-2$ graphes $\Delta_{j i}^{\prime}$.

Le même résultat est valable pour $\Delta_{i j}^{\prime}$.

Pour représenter la famille des graphes qu'on vient de définir, on notera :

$$
\Delta_{j i}^{\prime} \rightarrow_{i}^{\text {prop }} \Delta \quad\left(\text { resp. } \Delta_{i j}^{\prime} \rightarrow_{i}^{\text {prop }} \Delta\right) \text {. }
$$


Proposition 5.2 (L'opérateur $\partial$ sur les graphes). Soit $\delta=\sum_{\Delta} a_{\Delta} \Delta$ une combinaison linéaire symétrique homogène de graphes aériens vectoriels. Alors

avec

$$
\partial C_{\delta}=\sum_{\Delta} a_{\Delta} C_{\partial \Delta}=C_{\partial \delta}
$$

$$
\partial \Delta=-\sum_{j \neq i} \varepsilon_{\{|\operatorname{Deb}(0)|, \ldots,|\operatorname{Deb}(n)|\}}(j, 0, \ldots \hat{\jmath} \ldots, n) \sum_{\Delta_{j i}^{\prime} \rightarrow_{i}^{\text {prop }} \Delta} \Delta_{j i}^{\prime} .
$$

On peut écrire $\partial \Delta$ autrement:

$$
\begin{aligned}
\partial \Delta=- & \sum_{i<j}\left(\varepsilon_{|\operatorname{Deb}|}(j, 0, \ldots \hat{\jmath} \ldots, n) \sum_{\Delta_{j i}^{\prime} \rightarrow_{i}^{\text {prop }} \Delta} \Delta_{j i}^{\prime}\right. \\
& +\left((1-|\operatorname{Deb}(j)|) \varepsilon_{|\operatorname{Deb}|}(i, 0, \ldots \hat{\imath} \ldots, n)-|\operatorname{Deb}(j)| \varepsilon_{|\operatorname{Deb}|}(j, 0, \ldots \hat{\jmath} \ldots, n)\right) \\
& \left.\times \sum_{\Delta_{i j}^{\prime} \rightarrow{ }_{i}^{\text {prop }} \Delta} \Delta_{i j}^{\prime}\right) .
\end{aligned}
$$

Démonstration. On sait que $\left(\partial C_{\delta}\right)\left(\alpha_{0}, \ldots, \alpha_{n}\right)=(\mathrm{I})+(\mathrm{II})-(\mathrm{III})$, où

$$
\begin{aligned}
(\mathrm{I}) & =\sum_{j=0}^{n} \varepsilon_{|\alpha|}(j, 0 \ldots \hat{\jmath} \ldots n)(-1)^{\left|C_{\delta}\right|\left(\left|\alpha_{j}\right|-1\right)} \nabla_{\alpha_{j}} C_{\delta}\left(\alpha_{0}, \ldots \widehat{\alpha}_{j} \ldots, \alpha_{n}\right), \\
(\mathrm{II}) & =\sum_{i=0}^{n}(-1)^{\left|C_{\delta}\right|} \varepsilon_{|\alpha|}(0 \ldots \hat{\imath} \ldots n, i) \nabla_{C_{\delta}\left(\alpha_{0}, \ldots \widehat{\alpha}_{i} \ldots, \alpha_{n}\right)} \alpha_{i}, \\
(\mathrm{III}) & =\sum_{i \neq j} \varepsilon_{|\alpha|}(j, i, 0, \ldots \hat{\imath} \ldots \hat{\jmath} \ldots n) C_{\delta}\left(\nabla_{\alpha_{j}} \alpha_{i}, \alpha_{0}, \ldots \widehat{\alpha_{i}} \ldots \widehat{\alpha}_{j} \ldots, \alpha_{n}\right) .
\end{aligned}
$$

Puisque la cohomologie de $C_{\delta}$ est déterminée par sa composante dans les fonctions ([Arnal et al. 2005], Proposition 5.2), on ne regarde que les termes qui sont des opérateurs 0 -différentiels (des fonctions) dans cette expression.

Les termes de la première somme n'apparaissent que si $\alpha_{j}$ est un champ de vecteurs $\alpha_{j}=\sum_{\ell} \alpha_{j}^{\ell} \partial_{\ell}$. On a

$$
(\mathrm{I})=\sum_{j \neq i} \varepsilon_{|\alpha|}(j, 0, \ldots \hat{\jmath} \ldots, n) \sum_{\ell} \alpha_{j}^{\ell} C_{\delta}\left(\alpha_{0}, \ldots \widehat{\alpha}_{j} \ldots, \partial_{\ell} \alpha_{i}, \ldots, \alpha_{n}\right)
$$

Dans le second terme, $\left|C_{\delta}\right|$ est le nombre $|\delta|$ de flèches des graphes de $\delta$. En revenant à la définition de $C_{\delta}$, ces termes apparaissent lorsque $C_{\delta}\left(\alpha_{0}, \ldots \widehat{\alpha}_{i} \ldots, \alpha_{n}\right)$ est un champ de vecteurs. Pour chaque $\Delta$ de $\delta$, on choisit un sommet $j$ tel que $\operatorname{Deb}\left(\alpha_{j}\right)=0$. Alors : 


$$
\begin{aligned}
& C_{\Delta}\left(\alpha_{0}, \ldots \widehat{\alpha}_{i} \ldots, \alpha_{n}\right) \\
& =\sum_{\left(\Gamma, \mathscr{O}_{\Gamma}\right) \supset(\Delta, \mathcal{O})} \varepsilon\left(\mathscr{O}_{\Gamma}, \mathscr{O}_{0}\right) \sum_{j, \ell} B_{\left(\Gamma, \mathscr{O}_{\Gamma}\right)}\left(\alpha_{0}, \ldots, \alpha_{j}^{\ell}, \ldots \widehat{\alpha}_{i} \ldots, \alpha_{n}\right) \partial_{\ell} \\
& =\sum_{\left(\Gamma, \bigcirc_{\Gamma}\right) \supset(\Delta, \mathcal{C})} \varepsilon_{|\alpha|}(0, \ldots \hat{\imath} \ldots \hat{\jmath} \ldots, n, j) \sum_{j, \ell} B_{\left(\Gamma, O_{\Gamma}\right)}\left(\alpha_{0}, \ldots, \alpha_{j}^{\ell}, \ldots \widehat{\alpha}_{i} \ldots, \alpha_{n}\right) \partial_{\ell} .
\end{aligned}
$$

Donc on obtient puisqu'ici $\left|\alpha_{i}\right|=0$,

$$
\begin{aligned}
(\mathrm{II}) & =\sum_{j \neq i}(-1)^{|\delta|} \varepsilon_{|\alpha|}(0, \ldots \hat{\imath} \ldots \hat{\jmath} \ldots, n, j) \sum_{\ell} C_{\delta}\left(\alpha_{0}, \ldots, \alpha_{j}^{\ell}, \ldots \widehat{\alpha}_{i} \ldots, \alpha_{n}\right) \partial_{\ell} \alpha_{i} \\
& =\sum_{j \neq i} \varepsilon_{|\alpha|}(j, 0, \ldots \hat{\jmath} \ldots, n) \sum_{\ell} C_{\delta}\left(\alpha_{0}, \ldots, \alpha_{j}^{\ell}, \ldots \widehat{\alpha}_{i} \ldots, \alpha_{n}\right) \partial_{\ell} \alpha_{i} \\
& =\sum_{j \neq i} \varepsilon_{|\alpha|}(j, 0, \ldots \hat{\jmath} \ldots, n) \sum_{\ell} C_{\delta}(\alpha_{0}, \ldots, \underbrace{\alpha_{j}^{\ell}}_{(i)}, \ldots \widehat{\alpha}_{j} \ldots, \alpha_{n}) \partial_{\ell} \alpha_{i} .
\end{aligned}
$$

Enfin la dernière somme s'écrit :

$$
\begin{aligned}
(\mathrm{III}) & =\sum_{i \neq j} \varepsilon_{|\alpha|}(j, i, 0, \ldots \hat{\imath} \ldots \hat{\jmath} \ldots, n) C_{\delta}\left(\nabla_{\alpha_{j}} \alpha_{i}, \alpha_{0}, \ldots \widehat{\alpha_{i}} \ldots \widehat{\alpha}_{j} \ldots, \alpha_{n}\right) \\
& =\sum_{i \neq j} \varepsilon_{|\alpha|}(j, 0, \ldots \hat{\jmath} \ldots, n) \sum_{\ell} C_{\delta}(\alpha_{0}, \ldots, \underbrace{\alpha_{j}^{\ell} \partial_{\ell} \alpha_{i}}_{(i)}, \ldots \widehat{\alpha}_{j} \ldots, \alpha_{n}) .
\end{aligned}
$$

Appliquons la règle de Leibniz pour les dérivations multiples dans le terme (III). On obtient pour chaque $\Delta$ de $\delta$ :

$$
\begin{aligned}
C_{\Delta} & (\alpha_{0}, \ldots, \underbrace{\alpha_{j}^{\ell} \partial_{\ell} \alpha_{i}}_{(i)}, \ldots \widehat{\alpha}_{j} \ldots, \alpha_{n}) \\
& =\left(\prod_{t \notin\{i, j\}} \partial_{\operatorname{Fin}(t)} \alpha_{t}^{\operatorname{Deb}(t)}\right) \partial_{\operatorname{Fin}(i)}\left(\alpha_{j}^{\ell} \partial_{\ell} \alpha_{i}^{\operatorname{Deb}(i)}\right) \\
& =\left(\prod_{t \notin\{i, j\}} \partial_{\operatorname{Fin}(t)} \alpha_{t}^{\operatorname{Deb}(t)}\right)\left(\sum_{A \subset \operatorname{Fin}(i)} \partial_{A} \alpha_{j}^{\ell} \partial_{\{\ell\} \cup\{\operatorname{Fin}(i) \backslash A\}} \alpha_{i}^{\operatorname{Deb}(i)}\right) .
\end{aligned}
$$

La somme (I) correspond exactement au cas $A=\varnothing$, la somme (II) au cas $A=$ Fin $(i)$. Il y a simplification. Posons, comme précédemment, $\partial C_{\Delta}=-\sum_{j \neq i} \varepsilon_{|\operatorname{Deb}|}(j, 0, \ldots \hat{\jmath} \ldots, n) \partial_{j i} C_{\Delta}=-\sum_{j \neq i} \varepsilon_{|\operatorname{Deb}|}(j, 0, \ldots \hat{\jmath} \ldots, n) C_{\partial_{j i} \Delta}$, où $\mid$ Deb $\mid$ est défini page 271. On peut écrire : 
$\underline{\operatorname{Si} \operatorname{Deb}(i)=\operatorname{Fin}(i)=\varnothing}$

$$
\partial_{j i} \Delta=\Delta_{j i}^{\prime}=\sum_{\Delta_{j i}^{\prime} \rightarrow_{i}^{\text {prop }} \Delta} \Delta_{j i}^{\prime} .
$$

$\underline{\operatorname{Si} \operatorname{Deb}(i)=\varnothing \text { et }|\operatorname{Fin}(i)|>1}$

$$
\partial_{j i} C_{\Delta}=\left(\prod_{t \notin\{i, j\}} \partial_{\operatorname{Fin}(t)} \alpha_{t}^{\operatorname{Deb}(t)}\right)\left(\sum_{\substack{A \subset \operatorname{Fin}(i) \\ A \neq \varnothing \\ A \neq \operatorname{Fin}(i)}} \partial_{A} \alpha_{j}^{\ell} \partial_{\{\ell\} \cup\{\operatorname{Fin}(i) \backslash A\}} \alpha_{i}\right)=\sum_{\Delta_{j i}^{\prime} \rightarrow_{i}^{\mathrm{prop}} \Delta} C_{\Delta_{j i}^{\prime}} .
$$

$\underline{\operatorname{Si} \operatorname{Deb}(i) \neq \varnothing \text { et } \operatorname{Fin}(i) \neq \varnothing}$

$$
\begin{aligned}
\partial_{j i} C_{\Delta} & =\left(\prod_{t \notin\{i, j\}} \partial_{\operatorname{Fin}(t)} \alpha_{t}^{\operatorname{Deb}(t)}\right)\left(\sum_{\substack{A \subset \operatorname{Fin}(i) \\
A \neq \varnothing}} \partial_{A} \alpha_{j}^{\ell} \partial_{\{\ell\} \cup\{\operatorname{Fin}(i) \backslash A\}} \alpha_{i}^{\operatorname{Deb}(i)}\right) \\
& =\sum_{\Delta_{j i}^{\prime} \rightarrow \rightarrow_{i}^{\text {prop }} \Delta} C_{\Delta_{j i}^{\prime}} .
\end{aligned}
$$

\section{$\underline{\operatorname{Si}|\operatorname{Deb}(i)|+|\operatorname{Fin}(i)|=1}$}

$$
\partial_{j i} \Delta=0=\sum_{\Delta_{j i}^{\prime} \rightarrow{ }_{i}^{\text {prop }} \Delta} \Delta_{j i}^{\prime} .
$$

Finalement on a donc bien :

$$
\partial \Delta=-\sum_{j \neq i} \varepsilon_{|\operatorname{Deb}|}(j, 0, \ldots \hat{\jmath} \ldots, n) \sum_{\Delta_{j i}^{\prime} \rightarrow \rightarrow_{i}^{\text {prop }} \Delta} \Delta_{j i}^{\prime} .
$$

D'autre part, on peut regrouper les termes de (III) autrement :

$$
\begin{aligned}
(\mathrm{III})=\sum_{i<j} \varepsilon_{|\alpha|}(j, i, 0, \ldots \hat{\imath} \ldots \hat{\jmath} \ldots, n) C_{\delta}\left(\nabla_{\alpha_{j}} \alpha_{i}, \alpha_{0}, \ldots \widehat{\alpha}_{i} \ldots \widehat{\alpha}_{j} \ldots, \alpha_{n}\right) \\
\quad+\sum_{i<j} \varepsilon_{|\alpha|}(i, j, 0, \ldots \hat{\imath} \ldots \hat{\jmath} \ldots, n) C_{\delta}\left(\nabla_{\alpha_{i}} \alpha_{j}, \alpha_{0}, \ldots \widehat{\alpha}_{i} \ldots \widehat{\alpha}_{j} \ldots, \alpha_{n}\right)
\end{aligned}
$$

ou

$$
\begin{aligned}
& \text { (III) }=\sum_{i<j} \varepsilon_{|\alpha|}(j, 0, \ldots \hat{\jmath} \ldots, n) \sum_{\ell} C_{\delta}(\alpha_{0}, \ldots, \underbrace{\alpha_{j}^{\ell} \partial_{\ell} \alpha_{i}}_{(i)}, \ldots \widehat{\alpha}_{j} \ldots, \alpha_{n}) \\
& +\sum_{i<j}(-1)^{\left|\alpha_{i}\right|\left|\alpha_{j}\right|} \varepsilon_{|\alpha|}(j, i, 0, \ldots \hat{\imath} \ldots \hat{\jmath} \ldots, n) \\
& \quad \times \sum_{\ell} C_{\delta}\left(\alpha_{i}^{\ell} \partial_{\ell} \alpha_{j}, \alpha_{0}, \ldots \widehat{\alpha}_{i} \ldots \widehat{\alpha}_{j} \ldots, \alpha_{n}\right) .
\end{aligned}
$$

On transforme le second terme ainsi : 
Si $\left|\alpha_{j}\right|=1$, ce terme est :

$$
-\varepsilon_{|\alpha|}(j, 0, \ldots \hat{\jmath} \ldots, n) \sum_{\ell} C_{\delta}(\alpha_{0}, \ldots, \underbrace{\alpha_{i}^{\ell} \partial_{\ell} \alpha_{j}}_{(i)}, \ldots \widehat{\alpha}_{j} \ldots, \alpha_{n}) .
$$

Si $\left|\alpha_{j}\right|=0$, ce terme est :

$$
\varepsilon_{|\alpha|}(i, 0, \ldots \hat{\imath} \ldots, n) \sum_{\ell} C_{\delta}(\alpha_{0}, \ldots, \underbrace{\alpha_{i}^{\ell} \partial_{\ell} \alpha_{j}}_{(i)}, \ldots \widehat{\alpha}_{j} \ldots, \alpha_{n}) .
$$

En regroupant les deux cas, on obtient le facteur annoncé :

$$
\begin{array}{r}
\left(\left(1-\left|\alpha_{j}\right|\right) \varepsilon_{|\alpha|}(i, 0, \ldots \hat{\imath} \ldots, n)-\left|\alpha_{j}\right| \varepsilon_{|\alpha|}(j, 0, \ldots \hat{\jmath} \ldots, n)\right) \\
\qquad \sum_{\ell} C_{\delta}(\alpha_{0}, \ldots, \underbrace{\alpha_{i}^{\ell} \partial_{\ell} \alpha_{j}}_{(i)}, \ldots \widehat{\alpha}_{j} \ldots, \alpha_{n}) .
\end{array}
$$

De même pour (I) et (II), on obtient

$$
\begin{aligned}
&(\mathrm{I})=\sum_{i<j}\left(\varepsilon_{|\alpha|}(j, 0, \ldots \hat{\jmath} \ldots, n) \sum_{\ell} \alpha_{j}^{\ell}\right. C_{\delta}(\alpha_{0}, \ldots, \underbrace{\partial_{\ell} \alpha_{i}}_{(i)}, \ldots \widehat{\alpha_{j}} \ldots, \alpha_{n}) \\
&+\left(\left(1-\left|\alpha_{j}\right|\right) \varepsilon_{|\alpha|}(i, 0, \ldots \hat{\imath} \ldots, n)-\left|\alpha_{j}\right| \varepsilon_{|\alpha|}(j, 0, \ldots \hat{\jmath} \ldots, n)\right) \\
&\left.\quad \times \sum_{\ell} \alpha_{i}^{\ell} C_{\delta}(\alpha_{0}, \ldots, \underbrace{\partial_{\ell} \alpha_{j}}_{(i)}, \ldots \widehat{\alpha_{j}} \ldots, \alpha_{n})\right)
\end{aligned}
$$

et

$$
\begin{aligned}
(\mathrm{II})=\sum_{i<j} \varepsilon_{|\alpha|}(j, 0, \ldots \hat{\jmath} \ldots, n) \sum_{\ell} C_{\delta}(\alpha_{0}, \ldots, \underbrace{\alpha_{j}^{\ell}}_{(i)}, \ldots \widehat{\alpha_{j}} \ldots, \alpha_{n}) \partial_{\ell} \alpha_{i} \\
\\
\quad+\varepsilon_{|\alpha|}(i, 0, \ldots \hat{\imath} \ldots, n) \sum_{\ell} C_{\delta}(\alpha_{0}, \ldots, \underbrace{\alpha_{i}^{\ell}}_{(i)}, \ldots \widehat{\alpha}_{j} \ldots, \alpha_{n}) \partial_{\ell} \alpha_{j} .
\end{aligned}
$$

En faisant le même calcul que ci-dessus, on simplifie les termes qui se correspondent dans (I), (II) et (III) et on obtient la formule annoncée.

Remarque 5.3. On a retrouvé le cobord défini dans [Arnal et al. 2005]. En particulier, si $\delta$ est symétrique, $\partial \delta$ est aussi symétrique.

On pose bien entendu

Définition 5.4 (Espaces de cohomologie sur les graphes). Une combinaison linéaire symétrique $\delta$ de graphes vectoriels aériens est un cocycle si $\partial \delta=0$, un cobord s'il existe une combinaison linéaire symétrique $\beta$ telle que $\delta=\partial \beta$. L'espace $Z^{n}$ des $n$-cocycles est l'espace des combinaisons symétriques de graphes vectoriels ayant $n$ sommets et qui sont des cocycles. L'espace $B^{n}$ des $n$-cobords est l'espace des combinaisons de graphes qui sont les cobords de combinaisons symétriques de graphes ayant $n-1$ sommets. Le $n^{\llbracket m e}$ espace de cohomologie des graphes $H^{n}$ est le quotient de l'espace $Z^{n}$ par l'espace $B^{n}$. 


\section{Symbole d'un graphe}

Soit $\Delta$ un graphe aérien vectoriel de sommets numérotés $(1, \ldots, n)$. On peut distinguer six classes de sommets $i$ :

Classe 1 : les sommets $i$ tels que $|\operatorname{Fin}(i)|>1$ et $|\operatorname{Deb}(i)|=0$. On appellera ordre de $i$ le symbole $r_{i}$ où $r_{i}=|\operatorname{Fin}(i)|$.

Classe 2 : les sommets $i$ tels que $|\operatorname{Fin}(i)|>1$ et $|\operatorname{Deb}(i)|=1$. On appellera ordre de $i$ le symbole $r_{i}^{+}$où $r_{i}=\mid$ Fin $(i) \mid$.

Classe 3 : les sommets $i$ tels que $|\operatorname{Fin}(i)|=1$ et $|\operatorname{Deb}(i)|=1$. On appellera ordre de $i$ le symbole $1^{+}$.

Classe 4 : les sommets $i$ tels que $|\operatorname{Fin}(i)|=1$ et $|\operatorname{Deb}(i)|=0$. On appellera ordre de $i$ le symbole 1 .

Classe 5 : les sommets $i$ tels que $|\operatorname{Fin}(i)|=0$ et $|\operatorname{Deb}(i)|=0$. On appellera ordre de $i$ le symbole 0 .

Classe 6 : les sommets $i$ tels que $|\operatorname{Fin}(i)|=0$ et $|\operatorname{Deb}(i)|=1$. On appellera ordre de $i$ le symbole $0^{-}$.

On ordonne les ordres des sommets en posant :

$$
r_{i}>r_{j}^{+}>1^{+}>1>0>0^{-} \quad \text { et } \quad r_{i}^{+} \geq r_{i^{\prime}}^{+} \Longleftrightarrow r_{i} \geq r_{i^{\prime}} .
$$

L'ordre $\mathcal{O}(\Delta)$ d'un graphe $\Delta$ est le mot formé par les ordres de ses sommets :

$$
\mathcal{O}(\Delta)=(\mathcal{O}(1), \ldots, \mathcal{O}(n)) .
$$

On ordonne les ordres des graphes en utilisant l'ordre lexicographique, en respec$\operatorname{tant}(6-1)$.

Si $\delta=\sum_{\Delta} a_{\Delta} \Delta$ est une combinaison linéaire symétrique de graphes vectoriels, on définit l'ordre de $\delta$ par :

$$
\mathcal{O}(\delta)=\max \left\{\mathcal{O}(\Delta), a_{\Delta} \neq 0\right\}
$$

et on appellera symbole de $\delta$ la combinaison linéaire non symétrique :

$$
\sigma_{\delta}=\sum_{\substack{\Delta \\ \mathcal{O}(\Delta)=\mathcal{O}(\delta)}} a_{\Delta} \Delta .
$$

Comme $\delta$ est symétrique, son ordre a la forme :

$\mathcal{O}(\delta)=(r_{1}, \ldots, r_{k_{0}-1}, r_{k_{0}}^{+}, \ldots, r_{k_{1}-1}^{+}, \underbrace{1^{+}}_{\left(k_{1}\right)}, \ldots, 1^{+}, \underbrace{1}_{\left(k_{2}\right)}, \ldots, 1, \underbrace{0}_{\left(k_{3}\right)}, \ldots, 0, \underbrace{0^{-}}_{\left(k_{4}\right)}, \ldots, 0^{-})$

avec

$$
r_{1} \geq r_{2} \cdots \geq r_{k_{0}-1}, r_{k_{0}}^{+} \geq \cdots \geq r_{k_{1}-1}^{+} .
$$

(On peut avoir $k_{0}=1$ ou $k_{1}=k_{0}$, etc.) 
Proposition 6.1 (Le symbole de $\partial \delta$ ). Soit $\delta$ une combinaison linéaire symétrique de graphes aériens vectoriels, d'ordre

$$
\mathcal{O}(\delta)=(r_{1}, \ldots, r_{k_{0}-1}, r_{k_{0}}^{+}, \ldots, r_{k_{1}-1}^{+}, \underbrace{1^{+}}_{\left(k_{1}\right)}, \ldots, 1^{+}, \underbrace{1}_{\left(k_{2}\right)}, \ldots, 1, \underbrace{0}_{\left(k_{3}\right)}, \ldots, 0, \underbrace{0^{-}}_{\left(k_{4}\right)}, \ldots, 0^{-}) .
$$

Alors chaque graphe $\Delta^{\prime}$ apparaissant dans $\partial \delta$ est d'ordre au plus:

$$
\begin{aligned}
& \mathcal{O}(\delta) \oplus 1^{+} \\
& =(r_{1}, \ldots, r_{k_{0}-1}, r_{k_{0}}^{+}, \ldots, r_{k_{1}-1}^{+}, \underbrace{1^{+}}_{\left(k_{1}\right)}, \ldots, 1^{+}, \underbrace{1}_{\left(k_{2}+1\right)}, \ldots, 1, \underbrace{0}_{\left(k_{3}+1\right)}, \ldots, 0, \underbrace{0^{-}}_{\left(k_{4}+1\right)}, \ldots, 0^{-}) .
\end{aligned}
$$

Si $\mathcal{O}(\partial \delta)=\mathcal{O}(\delta) \oplus 1^{+}$, alors le symbole de $\partial \delta$ est:

$$
\begin{aligned}
& \sigma_{\partial \delta}=-\sum_{\Delta \in \sigma_{\delta}} a_{\Delta}\left(\sum_{\substack{i<j \\
0 \leq i<k_{0} \\
k_{1} \leq j \leq k_{2}}} \varepsilon_{|\mathrm{Deb}|}(j, 0, \ldots \hat{\jmath} \ldots, n) \sum_{\Delta_{j i}^{\prime} \rightarrow \rightarrow_{i}^{\text {prop }} \Delta} \Delta_{j i}^{\prime}\right. \\
& \quad+\sum_{\substack{i<j \\
k_{0} \leq i<k_{1} \\
k_{1} \leq j \leq k_{2}}} \varepsilon_{|\operatorname{Deb}|}(j, 0, \ldots \hat{\jmath} \ldots, n)\left(\sum_{\Delta_{j i}^{\prime} \rightarrow i_{i}^{\text {prop }} \Delta} \Delta_{j i}^{\prime}-\sum_{\Delta_{i j}^{\prime} \rightarrow i_{i}^{\text {prop }} \Delta} \Delta_{i j}^{\prime}\right) \\
&\left.+\sum_{\substack{i<j \\
k_{1} \leq i<k_{2} \\
k_{1} \leq j \leq k_{2}}} \varepsilon_{|\operatorname{Deb}|}(j, 0, \ldots \hat{\jmath} \ldots, n)\left(\sum_{\Delta_{j i}^{\prime} \rightarrow{ }_{i}^{\text {prop }} \Delta} \Delta_{j i}^{\prime}-\sum_{\Delta_{i j}^{\prime} \rightarrow \rightarrow_{i}^{\text {prop }} \Delta} \Delta_{i j}^{\prime}\right)\right) .
\end{aligned}
$$

Démonstration. Si $\Delta$ est un graphe de $\delta$ qui n'apparaît pas dans le symbole $\sigma_{\delta}$, alors les ordres des graphes $\Delta_{i j}^{\prime}$ et $\Delta_{j i}^{\prime}$ qui se contractent sur $\Delta$ sont tous strictement plus petits que $O(\delta) \oplus 1^{+}$. Regardons donc seulement les $\Delta$ de $\sigma_{\delta}$.

Fixons un couple $(i, j)$ avec $i<j$. Il est clair que dans la décomposition de $\partial \Delta$, les graphes $\Delta^{\prime}=\Delta_{j i}^{\prime}$ ou $\Delta^{\prime}=\Delta_{i j}^{\prime}$ sont d'ordre :

Si $0 \leq i<k_{0}$ :

$$
\mathcal{O}\left(\Delta_{j i}^{\prime}\right)=(r_{1}, \ldots, \underbrace{\left(r_{i}-k+1\right)}_{(i)}, \ldots, \underbrace{k^{+}}_{(j)}, \ldots) \quad\left(1 \leq k<r_{i}\right) .
$$

Donc $\mathcal{O}\left(\Delta_{j i}^{\prime}\right) \leq \mathcal{O}(\delta) \oplus 1^{+}$et l'égalité n'est vraie que si $k=1$ et $k_{1} \leq j \leq k_{2}$. Il y a $r_{i}$ graphes $\Delta_{j i}^{\prime}$ dans ce cas. D'autre part :

$$
\mathcal{O}\left(\Delta_{i j}^{\prime}\right)=(r_{1}, \ldots, \underbrace{k^{+}}_{(i)}, \ldots, \underbrace{\left(r_{i}-k+1\right)}_{(j)}, \ldots) \quad\left(1 \leq k<r_{i}\right) .
$$

Donc $\mathcal{O}\left(\Delta_{i j}^{\prime}\right)<\mathcal{O}(\delta) \oplus 1^{+}$. Il n'y a aucun graphe $\Delta_{i j}^{\prime}$, dans ce cas, qui apparaît dans $\sigma_{\partial \delta}$ 
Si $k_{0} \leq i<k_{1}$ :

$$
\mathcal{O}\left(\Delta_{j i}^{\prime}\right)=(r_{1}, \ldots, \underbrace{\left(r_{i}-k+1\right)^{+}}_{(i)}, \ldots, \underbrace{k^{+}}_{(j)}, \ldots) \quad\left(1 \leq k \leq r_{i}\right) .
$$

Donc $\mathcal{O}\left(\Delta_{j i}^{\prime}\right) \leq \mathcal{O}(\delta) \oplus 1^{+}$et l'égalité n'est vraie que si $k=1$ et $k_{1} \leq j \leq k_{2}$. Il y a $r_{i}$ graphes $\Delta_{j i}^{\prime}$ dans ce cas. D'autre part :

$$
\mathcal{O}\left(\Delta_{i j}^{\prime}\right)=(r_{1}, \ldots, \underbrace{k^{+}}_{(i)}, \ldots, \underbrace{\left(r_{i}-k+1\right)^{+}}_{(j)}, \ldots) \quad\left(1 \leq k \leq r_{i}\right) .
$$

Donc $\mathcal{O}\left(\Delta_{i j}^{\prime}\right) \leq \mathcal{O}(\delta) \oplus 1^{+}$et l'égalité n'est vraie que si $k=r_{i}$ et $k_{1} \leq j \leq k_{2}$. Il y a un seul graphe $\Delta_{i j}^{\prime}$ dans ce cas.

Si $k_{1} \leq i<k_{2}$ :

$$
O\left(\Delta^{\prime}\right)=(r_{1}, \ldots, \underbrace{1^{+}}_{(i)}, \ldots, \underbrace{1^{+}}_{(j)}, \ldots)
$$

Donc pour et seulement pour $i<j \leq k_{2}, \mathcal{O}\left(\Delta^{\prime}\right)=\mathcal{O}(\delta) \oplus 1^{+}$et il y a un seul graphe $\Delta_{j i}^{\prime}$ et un seul graphe $\Delta_{i j}^{\prime}$ dans ce cas.

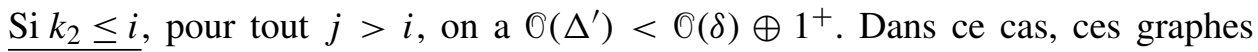
n'apparaîssent pas dans $\sigma_{\partial \delta}$.

Définissons maintenant l'opérateur d'homotopie.

Définition 6.2 (L'homotopie). Soit $\Delta$ un graphe vectoriel de sommets $(0, \ldots, n)$, ayant des sommets $i$ tels que $|\operatorname{Deb}(i)|=|\operatorname{Fin}(i)|=1$ (des sommets $i$ d'ordre $1^{+}$). On définit l'homotopie $h$ comme l'application qui transforme le graphe $\Delta$ en le graphe $h(\Delta)$ défini ainsi :

On considère $i_{0}$, le plus grand des indices $i$ d'ordre $1^{+}$. La flèche issue de $i_{0}$ est $i_{0} a$. Le graphe $h(\Delta)$ est le graphe de sommets $\left(0, \ldots \hat{i_{0}} \ldots, n\right)$ obtenu en comprimant la flèche $i_{0} a$ de $\Delta$ et en identifiant les sommets $i_{0}$ et $a$ au sommet $a$.

Si $\Delta$ n'a pas de sommets d'ordre $1^{+}$, on pose $h(\Delta)=0$.

On prolonge $h$ linéairement à l'espace des combinaisons linéaires de graphes.

Proposition 6.3 (Symbole et homotopie). Soit $\delta$ une combinaison linéaire symétrique de graphes vectoriels d'ordre

$$
\mathcal{O}(\delta)=(r_{1}, \ldots, r_{k_{0}-1}, r_{k_{0}}^{+}, \ldots, r_{k_{1}-1}^{+}, \underbrace{1^{+}}_{\left(k_{1}\right)}, \ldots, 1^{+}, \underbrace{1}_{\left(k_{2}\right)}, \ldots, 1, \underbrace{0}_{\left(k_{3}\right)}, \ldots, 0, \underbrace{0^{-}}_{\left(k_{4}\right)}, \ldots, 0^{-}) .
$$

Par abus de notations, on pose:

$$
\sigma_{\partial \delta}=\sum_{\substack{\Delta^{\prime} \in \partial \delta \\ \mathcal{O}\left(\Delta^{\prime}\right)=\mathcal{O}(\delta) \oplus 1^{+}}} a_{\Delta^{\prime}} \Delta^{\prime} \quad \text { si } \partial \delta=\sum_{\Delta^{\prime}} a_{\Delta^{\prime}} \Delta^{\prime} .
$$


Notons enfin $\delta\left(0, \ldots \widehat{k}_{2} \ldots, n\right)$ la combinaison linéaire de graphes $\Delta$ dont on a renuméroté les sommets en $\left(0, \ldots \widehat{k}_{2} \ldots, n\right)$. Alors

$$
\begin{aligned}
& h\left(\sigma_{\partial \delta}\right)\left(0, \ldots \widehat{k}_{2} \ldots, n\right)=\sigma_{\partial h\left(\sigma_{\delta}\right)}\left(0, \ldots \widehat{k}_{2} \ldots, n\right) \\
& \quad-\varepsilon_{|\mathrm{Deb}|}\left(k_{2}, 0, \ldots \widehat{k}_{2} \ldots, n\right)\left(\sum_{0 \leq i<k_{0}} r_{i}+\sum_{k_{0} \leq i<k_{1}}\left(r_{i}-1\right)\right) \sigma_{\delta}\left(0, \ldots \widehat{k}_{2} \ldots, n\right) .
\end{aligned}
$$

Démonstration. On reprend les notations de la proposition précédente. Le dernier $i$ d'ordre $1^{+}$dans $\partial \delta$ est $k_{2}$. On écrit donc :

$$
\begin{aligned}
& \sigma_{\partial \delta}=-\sum_{\Delta \in \sigma_{\delta}} a_{\Delta}\left(\sum_{\substack{i<j \\
0 \leq i<k_{0} \\
j=k_{2}}} \varepsilon_{|\mathrm{Deb}|}\left(k_{2}, 0, \ldots \widehat{k}_{2} \ldots, n\right) \sum_{\Delta_{k_{2} i}^{\prime} \rightarrow{ }_{i}^{\text {prop }} \Delta} \Delta_{k_{2} i}^{\prime}\right. \\
& +\sum_{\substack{i<j \\
0 \leq i<k_{0} \\
k_{1} \leq j<k_{2}}} \varepsilon_{|\operatorname{Deb}|}(j, 0, \ldots \hat{\jmath} \ldots, n) \sum_{\Delta_{j i}^{\prime} \rightarrow \rightarrow_{i}^{\text {prop }} \Delta} \Delta_{j i}^{\prime} \\
& +\sum_{\substack{i<j \\
k_{0} \leq i<k_{1} \\
j=k_{2}}} \varepsilon_{|\mathrm{Deb}|}\left(k_{2}, 0, \ldots \widehat{k}_{2} \ldots, n\right)\left(\sum_{\Delta_{k_{2} i}^{\prime} \rightarrow_{i}^{\text {prop }} \Delta} \Delta_{k_{2} i}^{\prime}-\sum_{\Delta_{i k_{2}}^{\prime} \rightarrow{ }_{i}^{\text {prop }} \Delta} \Delta_{i k_{2}}^{\prime}\right) \\
& +\sum_{\substack{i<j \\
k_{0} \leq i<k_{1} \\
k_{1} \leq j<k_{2}}} \varepsilon_{|\operatorname{Deb}|}(j, 0, \ldots \hat{\jmath} \ldots, n)\left(\sum_{\Delta_{j i}^{\prime} \rightarrow{ }_{i}^{\mathrm{prop}} \Delta} \Delta_{j i}^{\prime}-\sum_{\Delta_{i j}^{\prime} \rightarrow{ }_{i}^{\text {prop }} \Delta} \Delta_{i j}^{\prime}\right) \\
& +\sum_{\substack{i<j \\
k_{1} \leq i<k_{2} \\
j=k_{2}}} \varepsilon_{|\mathrm{Deb}|}\left(k_{2}, 0, \ldots \widehat{k}_{2} \ldots, n\right)\left(\sum_{\Delta_{k_{2} i}^{\prime} \rightarrow i}^{\text {prop }} \Delta \Delta_{k_{2} i}^{\prime}-\sum_{\Delta_{i k_{2}}^{\prime} \rightarrow{ }_{i}^{\text {prop }} \Delta} \Delta_{i k_{2}}^{\prime}\right) \\
& \left.+\sum_{\substack{i<j \\
k_{1} \leq i<k_{2}}} \varepsilon_{|\mathrm{Deb}|}(j, 0, \ldots \hat{\jmath} \ldots, n)\left(\sum_{\Delta_{j i}^{\prime} \rightarrow \rightarrow_{i}^{\text {prop }} \Delta} \Delta_{j i}^{\prime}-\sum_{\Delta_{i j}^{\prime} \rightarrow \rightarrow_{i}^{\text {prop }} \Delta} \Delta_{i j}^{\prime}\right)\right) . \\
& k_{1} \leq j<k_{2}
\end{aligned}
$$

Appliquons $h$, on obtient :

$$
\begin{aligned}
h\left(\sigma_{\partial \delta}\right)=- & \sum_{\Delta \in \sigma_{\delta}} a_{\Delta}\left(\varepsilon_{|\mathrm{Deb}|}\left(k_{2}, 0, \ldots \widehat{k}_{2} \ldots, n\right)\right. \\
& \left.\times\left(\sum_{0 \leq i<k_{0}} r_{i}+\sum_{k_{0} \leq i<k_{1}}\left(r_{i}-1\right)+\sum_{k_{1} \leq i<k_{2}}(1-1)\right) \Delta\left(0, \ldots \widehat{k}_{2} \ldots, n\right)+\text { reste }\right) .
\end{aligned}
$$


Le reste est composé de termes de la forme $h\left(\Delta_{i j}^{\prime}\right)$ ou $h\left(\Delta_{j i}^{\prime}\right)$ avec $i<j<k_{2}$. Par construction, $h$ étant la compression de la flèche issue du sommet $k_{2}$ dans $\Delta_{j i}^{\prime}$ (resp. $\Delta_{i j}^{\prime}$ ), il réalise une bijection entre les ensembles

$$
\left\{\Delta_{j i}^{\prime}, \Delta_{j i}^{\prime} \rightarrow_{i}^{\text {prop }} \Delta\right\} \stackrel{h}{\longrightarrow}\left\{B_{j i}^{\prime}, B_{j i}^{\prime} \rightarrow_{i}^{\text {prop }} h(\Delta)\right\}
$$

(resp. entre les ensembles

$$
\left.\left\{\Delta_{i j}^{\prime}, \Delta_{i j}^{\prime} \rightarrow_{i}^{\text {prop }} \Delta\right\} \stackrel{h}{\longrightarrow}\left\{B_{i j}^{\prime}, B_{i j}^{\prime} \rightarrow_{i}^{\text {prop }} h(\Delta)\right\}\right)
$$

où $B_{j i}^{\prime}$ (resp. $\left.B_{i j}^{\prime}\right)$ est un graphe de sommets numérotés $\left(0, \ldots \widehat{k}_{2} \ldots, n\right)$ et $h(\Delta)$ a pour sommets $\left(0, \ldots \hat{\jmath} \ldots \widehat{k}_{2} \ldots, n\right)$.

Dans le reste, chacun des termes correspondant est affecté du signe

$$
\varepsilon_{|\operatorname{Deb}|}(j, 0, \ldots \hat{\jmath} \ldots, n) .
$$

Ce signe coïncide avec le même signe calculé en supprimant l'indice $k_{2}$ :

$$
\varepsilon_{\left|\operatorname{Deb}\left(\Delta^{\prime}\right)\right|}(j, 0, \ldots \hat{\jmath} \ldots, n)=\varepsilon_{\left|\operatorname{Deb}\left(h\left(\Delta^{\prime}\right)\right)\right|}(j, 0, \ldots \hat{\jmath} \ldots, n) .
$$

Donc

$$
\text { reste }=-\sigma_{\partial h\left(\sigma_{\delta}\right)}\left(0, \ldots \hat{k_{2}} \ldots, n\right)
$$

\section{Cohomologie des graphes vectoriels}

Disons qu'un sommet $i$ d'un graphe vectoriel $\Delta$ est simple si $\mid$ Fin $(i) \mid \leq 1$.

Proposition 7.1 (Le symbole d'un cocycle ne contient que des sommets simples). Soit $\delta$ une combinaison linéaire symétrique de graphes aériens vectoriels. On suppose que $\delta$ est un cocycle $(\partial \delta=0)$. Alors il existe un cobord $\partial \beta$ tel que le symbole $\sigma_{\delta-\partial \beta}$ de $\delta-\partial \beta$ ne contient que des graphes $\Delta$ dont tous les sommets sont simples.

Démonstration. Puisque $\delta$ est un cocycle, $h\left(\sigma_{\partial \delta}\right)=0$. Dire que $\sigma_{\delta}$ contient au moins un graphe possédant un sommet non simple, c'est dire que dans $\mathcal{O}(\delta), k_{1}>0$. On a alors :

$$
0=-\varepsilon_{|\mathrm{Deb}|}\left(k_{2}, 0, \ldots \hat{k_{2}} \ldots, n\right)\left(\sum_{0 \leq i<k_{0}} r_{i}+\sum_{k_{0} \leq i<k_{1}}\left(r_{i}-1\right)\right) \sigma_{\delta}+\sigma_{\partial h\left(\sigma_{\delta}\right)} .
$$

Le coefficient $a$ de $\sigma_{\delta}$ est non nul. Puisque $\sigma_{\delta}$ n'est pas nul, on en déduit que $h\left(\sigma_{\delta}\right)$ n'est pas nul, donc $k_{2}>k_{1}$, il existe des sommets d'ordre $1^{+}$dans les graphes $\Delta$ de $\sigma_{\delta}$.

Posons

$$
\beta_{1}=-\frac{1}{a} S\left(h\left(\sigma_{\delta}\right)\right) .
$$


D'abord par construction, les graphes apparaissant dans $S\left(h\left(\sigma_{\delta}\right)\right)$ mais pas dans $h\left(\sigma_{\delta}\right)$ sont d'ordre strictement plus petit que les graphes apparaissant dans $h\left(\sigma_{\delta}\right)$, ou :

$$
\sigma_{h\left(\sigma_{\delta}\right)}=\sigma_{S\left(h\left(\sigma_{\delta}\right)\right)} .
$$

Ensuite on a vu que pour calculer le symbole de $\partial \delta$, on ne considérait que les graphes du symbole de $\delta$, donc :

$$
\sigma_{\partial h\left(\sigma_{\delta}\right)}=\sigma_{\partial S}\left(h\left(\sigma_{\delta}\right)\right)=-a \sigma_{\partial \beta_{1}}
$$

Alors,

$$
\sigma_{\partial \beta_{1}}=\sigma_{\delta} .
$$

Autrement dit $\delta_{1}=\delta-\partial \beta_{1}$ a un ordre strictement plus petit que $\mathcal{O}(\delta)$. Si le symbole de $\delta_{1}$ a des graphes avec des sommets non simples, on peut recommencer cette opération. Au bout d'un nombre fini d'étapes, on arrive sur une combinaison de graphes $\delta-\partial \beta$ dont le symbole ne contient que des sommets simples :

$$
\mathcal{O}(\delta-\partial \beta)=\left(1^{+}, \ldots, 1^{+}, 1, \ldots, 1,0, \ldots, 0,0^{-}, \ldots, 0^{-}\right) .
$$

Donc tous les graphes de $\delta-\partial \beta$ n'ont eux aussi que des sommets simples.

Définition 7.2 (Les roues). Une roue symétrique $R_{k}$ de longueur $k$ est le symétrisé de la roue simple qui est le graphe $\Delta_{k}$ ayant $k$ sommets $\{1, \ldots, k\}$ et les $k$ flèches $\{12,23, \ldots,(k-1) k, k 1\}$.

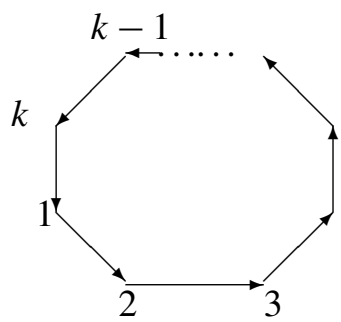

Lemme 7.3 (La cohomologie des roues). Les roues symétriques de longueur paire sont nulles, $R_{2 k}=0$.

Les roues impaires sont des cocycles $\partial R_{2 k+1}=0$ qui ne sont pas des cobords.

Démonstration. Soit $\Delta_{2 k}$ une roue simple de longueur paire. Soit $\sigma$ la permutation circulaire $\sigma=(1,2, \ldots, 2 k)$. Alors, $\varepsilon(\sigma)=\varepsilon_{\mid \text {Deb } \mid}(\sigma)=-1$ et $\sigma\left(\Delta_{2 k}\right)=\Delta_{2 k}$. Donc $R_{2 k}=S\left(\Delta_{2 k}\right)=0$.

Par contre les roues $R_{2 k+1}$ de longueur impaire ne sont pas nulles. En effet si on suppose la dimension de l'espace $\mathbb{R}^{d}$ assez grande, l'opérateur

$$
\begin{aligned}
& C_{R_{2 k+1}}\left(\alpha_{1}, \ldots, \alpha_{2 k+1}\right) \\
& =\frac{1}{(2 k+1) !} \sum_{\sigma \in S_{2 k+1}} \varepsilon(\sigma) \sum_{1 \leq t_{1} \ldots t_{2 k+1} \leq d} \partial_{t_{2 k+1}} \alpha_{\sigma(1)}^{t_{1}} \partial_{t_{1}} \alpha_{\sigma(2)}^{t_{2}} \ldots \partial_{t_{2 k}} \alpha_{\sigma(2 k+1)}^{t_{2 k+1}} .
\end{aligned}
$$


Cet opérateur est le cocycle non trivial $\zeta^{(2 k+1)}$ de [De Wilde et Lecomte 1983]. Comme il n'est pas nul, le graphe correspondant n'est pas nul non plus.

Soit $\Delta$ un graphe apparaissant dans la roue symétrique $R_{2 k+1}$. Les graphes $\Delta_{i j}^{\prime}$ et $\Delta_{j i}^{\prime}$ apparaissant dans $\partial \Delta$ sont tous des roues de longueurs $2 k+2$ (à l'ordre de leur sommets près). Comme $\partial R_{2 k+1}$ est symétrique, on a donc $\partial R_{2 k+1}=0$.

Supposons que $R_{2 k+1}=\partial \beta$. Il est clair que $\beta$ ne contient que des graphes ayant $2 k$ sommets tous d'ordres $1^{+}$. Donc $\beta$ est une combinaison linéaire de roues de longueur paire, $\beta=0$, ce qui est impossible. $R_{2 k+1}$ n'est pas un cobord.

Lemme 7.4 (Les graphes à roues). Si $\Delta$ est un graphe vectoriel dont toutes les composantes connexes sont des roues de longueurs impaires, alors le symétrisé de $\Delta$ est nul si deux composantes connexes ont la même longueur, sinon $S(\Delta)$ est au signe près le symétrisé du graphe:

$$
\Delta_{k_{1}} \wedge \Delta_{k_{2}} \wedge \cdots \wedge \Delta_{k_{p}}
$$

dont les flèches sont:

$$
\begin{aligned}
& \left\{12, \ldots,\left(k_{1}-1\right) k_{1}, k_{1} 1,\right. \\
& \quad\left(k_{1}+1\right)\left(k_{1}+2\right), \ldots,\left(k_{1}+k_{2}-1\right)\left(k_{1}+k_{2}\right),\left(k_{1}+k_{2}\right)\left(k_{1}+1\right), \ldots, \\
& \left.\quad\left(k_{1}+\cdots+k_{p-1}+1\right)\left(k_{1}+\cdots+k_{p-1}+2\right), \ldots,\left(k_{1}+\cdots+k_{p}\right)\left(k_{1}+\cdots+k_{p-1}+1\right)\right\}
\end{aligned}
$$

et $k_{1}<k_{2}<\cdots<k_{p}$.

Démonstration. Si deux composantes connexes de $\Delta$ sont des roues de même longueur $2 k+1$, de sommets numérotés $\left\{i_{1}, \ldots, i_{2 k+1}, j_{1}, \ldots, j_{2 k+1}\right\}$, alors la permutation

$$
\sigma=\left(i_{1}, j_{1}\right) \ldots\left(i_{2 k+1}, j_{2 k+1}\right)
$$

est impaire et laisse $\Delta$ invariant, donc le symétrisé de $\Delta$ est nul.

Il est clair que si les composantes connexes de $\Delta$ sont toutes des roues de longueurs impaires différentes, il existe une permutation des sommets de $\Delta$ qui le transforme en $\Delta_{k_{1}} \wedge \Delta_{k_{2}} \wedge \cdots \wedge \Delta_{k_{p}}$ avec $k_{1}<k_{2}<\cdots<k_{p}$. On notera abusivement :

$$
S(\Delta)=R_{k_{1}} \wedge R_{k_{2}} \wedge \cdots \wedge R_{k_{p}} .
$$

Lemme 7.5 (La cohomologie des graphes à roues). Tous les graphes à roues $R=$ $\bigwedge_{i=1}^{p} R_{2 k_{i}+1}$ sont des cocycles qui sont linéairement indépendants dans l'espace de cohomologie.

Démonstration. D'abord chacun de ces graphes est un cocycle, ensuite si une combinaison linéaire de ces graphes symétriques est un cobord $\left(\sum_{j} a_{j} R_{j}=\partial \beta\right)$, en se plaçant sur un espace $\mathbb{R}^{d}$ de dimension assez grande, on obtient une combinaison 
linéaire d'opérateurs

$$
\sum_{j} a_{j}\left(\bigwedge_{i=1}^{p_{j}} \zeta^{\left(2 k_{i}+1\right)}\right)
$$

qui est un cobord pour la cohomologie de Chevalley de l'algèbre de Lie des champs de vecteurs sur $\mathbb{R}^{d}$ à valeur dans les fonctions (les 0 -formes). Ceci n'est possible que si chaque $a_{j}$ est nul d'après [De Wilde et Lecomte 1983].

Définition 7.6 (Les lignes). La ligne symétrique $L_{\ell}$ de longueur $\ell$ est le symétrisé de la ligne simple $\Delta_{\ell}$, graphe de sommets $\{1, \ldots, \ell+1\}$ et de flèches $\{21,32, \ldots$, $(\ell+1) \ell\}$. Par convention, la ligne $L_{0}$ est le graphe à un sommet $\{1\}$ et sans flèche.

Lemme 7.7 (Cohomologie des lignes). Les lignes symétriques de longueur impaire $L_{2 \ell+1}$ sont des cobords. Le symétrisé d'un graphe contenant deux lignes de même longueur impaire est nul. Les lignes symétriques de longueur paire $L_{2 \ell}$ ne sont pas des cocycles, plus précisément, on a:

$$
\partial L_{2 \ell}=L_{2 \ell+1} .
$$

Démonstration. Prenons une ligne simple $\Delta_{\ell}$. Si $\ell>0$, dans le calcul de $\partial \Delta_{\ell}$, on éclate les $\ell-1$ sommets qui ne sont pas les extrémités de la ligne :

$$
\partial \Delta_{\ell}=-\sum_{i \neq j} \varepsilon_{|\mathrm{Deb}|}(j, 0, \ldots \hat{\jmath} \ldots, \ell+1) \sum_{\Delta_{j i}^{\prime} \rightarrow i_{i}^{\text {prop }} \Delta_{\ell}} \Delta_{j i}^{\prime} .
$$

Pour chaque couple $i \neq j$, il y a un seul graphe $\Delta_{j i}^{\prime}$. A l'ordre des sommets près, on obtient à chaque fois une ligne de longueur $\ell+1$.

On cherche donc dans le cobord de $L_{\ell}$ les lignes simples $\Delta_{\ell+1}$. Ces lignes ne peuvent provenir que de l'éclatement du sommet $i$ de la ligne simple $\Delta_{\ell}$ avec les sommets $\{0, \ldots l \hat{+} 1 \ldots, \ell+1\}$ et pour $j=i+1$. On obtient dans $\partial L_{\ell}$, donc,

$$
\begin{aligned}
-\left(\sum_{i=0}^{\ell} \varepsilon_{|\mathrm{Deb}|}(i+1,0, \ldots \widehat{\imath+1} \ldots, \ell+1)\right) \Delta_{\ell+1} & =-\left(\sum_{i=0}^{\ell}(-1)^{i}\right) \Delta_{\ell+1} \\
& =\left\{\begin{array}{cccc}
0 & \text { si } & \ell & \text { est impair, } \\
\Delta_{\ell+1} & \text { si } & \ell & \text { est pair. }
\end{array}\right.
\end{aligned}
$$

En symétrisant, on obtient :

$$
\partial L_{2 \ell+1}=0, \quad \partial L_{2 \ell}=L_{2 \ell+1} .
$$

Si $\Delta$ contient deux composantes connexes qui sont des lignes de même longueur impaire $2 \ell+1$, de sommets numérotés $i_{1}, \ldots, i_{2 \ell+2}$ et $j_{1}, \ldots, j_{2 \ell+2}$, la permutation

$$
\sigma=\left(i_{1}, j_{1}\right) \ldots\left(i_{2 \ell+2}, j_{2 \ell+2}\right)
$$


est telle que :

$$
\varepsilon_{|\mathrm{Deb}|}(\sigma)=-1 \text { et } \sigma(\Delta)=\Delta .
$$

Donc le symétrisé $S(\Delta)$ de $\Delta$ est nul.

Lemme 7.8 (Les graphes à lignes). Si $\Delta$ est un graphe dont toutes les composantes connexes sont des lignes, si le symétrisé $S(\Delta)$ de $\Delta$ n'est pas nul, c'est au signe près le symétrisé du graphe

$$
\left(\Delta_{0}^{k_{0}} \Delta_{2}^{k_{1}} \ldots \Delta_{2 \ell}^{k_{\ell}}\right) \Delta_{2 \ell_{1}+1} \wedge \Delta_{2 \ell_{2}+1} \wedge \ldots \wedge \Delta_{2 \ell_{q}+1} \quad\left(k_{0}, \ldots, k_{\ell} \in \mathbb{N}, \ell_{1}<\ell_{2}<\ldots<\ell_{q}\right) .
$$

Les sommets de ce graphe sont rangés dan l'ordre naturel; par exemple $\Delta_{2 \ell}^{k_{\ell}}$ désigne un graphe ayant $k_{\ell}(2 \ell+1)$ sommets formé d'une union de $k_{\ell}$ lignes de longueur $2 \ell$, en numérotant d'abord les sommets de la première ligne, puis ceux de la seconde, etc.

Démonstration. Si le symétrisé de $\Delta$ n'est pas nul, pour chaque longueur impaire, il y a au plus une composante connexe de cette longueur. Les composantes de longueur paire (y compris 0) peuvent apparaître plusieurs fois. Modulo une permutation des sommets, $\Delta$ est donc bien un produit de lignes comme annoncé.

Notons abusivement le symétrisé de ce graphe

$$
\left(L_{0}^{k_{0}} L_{2}^{k_{1}} \ldots L_{2 \ell}^{k_{\ell}}\right) L_{2 \ell_{1}+1} \wedge L_{2 \ell_{2}+1} \wedge \cdots \wedge L_{2 \ell_{q}+1} .
$$

Lemme 7.9 (La cohomologie des graphes à lignes). Considérons le graphe à lignes

$$
\left(\prod_{i=0}^{p} L_{2 i}^{k_{i}}\right)\left(\bigwedge_{j=1}^{q} L_{2 \ell_{j}+1}\right)
$$

Si pour chaque $i, k_{i} \neq 0$ implique qu'il existe $j$ tel que $i=\ell_{j}$, alors, il est, à la fois, un cocycle et un cobord

$$
\partial\left(\prod_{i=0}^{p} L_{2 i}^{k_{i}}\right)\left(\bigwedge_{j=1}^{q} L_{2 \ell_{j}+1}\right)=0
$$

Sinon

$$
\partial\left(\prod_{i=0}^{p} L_{2 i}^{k_{i}}\right)\left(\bigwedge_{j=1}^{q} L_{2 \ell_{j}+1}\right)=\sum_{r=0}^{p} k_{r}\left(\prod_{i \neq r} L_{2 i}^{k_{i}}\right) L_{2 r}^{k_{r}-1} L_{2 r+1} \wedge\left(\bigwedge_{j=1}^{q} L_{2 \ell_{j}+1}\right) \neq 0 .
$$

La cohomologie des graphes à lignes est triviale.

Démonstration. Il résulte des calculs précédents et du fait que les lignes de longueur paire peuvent permuter avec toutes les autres lignes sans changement de signe que :

$$
\partial\left(\prod_{i=0}^{p} L_{2 i}^{k_{i}}\right)\left(\bigwedge_{j=1}^{q} L_{2 \ell_{j}+1}\right)=\sum_{r=0}^{p} k_{r}\left(\left(\prod_{i \neq r} L_{2 i}^{k_{i}}\right) L_{2 r}^{k_{r}-1} L_{2 r+1} \wedge\left(\bigwedge_{j=1}^{q} L_{2 \ell_{j}+1}\right)\right) .
$$


Donc si pour chaque $i, k_{i} \neq 0$ implique qu'il existe $j \in\{1, \ldots, q\}$ tel que $i=\ell_{j}$, alors

$$
\partial\left(\prod_{i=0}^{p} L_{2 i}^{k_{i}}\right)\left(\bigwedge_{j=1}^{q} L_{2 \ell_{j}+1}\right)=0
$$

S'il existe $i$ tel que $k_{i}>0$ et il n'y a pas de $j$ tel que $i=\ell_{j}$, le graphe n'est pas un cocycle car si $r \neq s$,

$$
\left(\prod_{i \neq r} L_{2 i}^{k_{i}}\right) L_{2 r}^{k_{r}-1} L_{2 r+1} \wedge\left(\bigwedge_{j=1}^{q} L_{2 \ell_{j}+1}\right) \neq\left(\prod_{i \neq s} L_{2 i}^{k_{i}}\right) L_{2 s}^{k_{s}-1} L_{2 s+1} \wedge\left(\bigwedge_{j=1}^{q} L_{2 \ell_{j}+1}\right) .
$$

En particulier le graphe à lignes simples

$$
\left(\prod_{i=0}^{p-1} \Delta_{2 i}^{k_{i}}\right) \Delta_{2 p}^{k_{p}-1} \Delta_{2 p+1} \wedge\left(\bigwedge_{j=1}^{q} \Delta_{2 \ell_{j}+1}\right)
$$

n'apparaît qu'une seule fois. Le second membre n'est pas nul.

Si un graphe à lignes

$$
L=\left(\prod_{i=0}^{p} L_{2 i}^{k_{i}}\right)\left(\bigwedge_{j=1}^{q} L_{2 \ell_{j}+1}\right)
$$

est un cocycle, $2 \ell_{q}+1$ est la plus grande longueur des graphes apparaissant dans $L$ et si $p=\ell_{q}$, on a

$$
L=(-1)^{q-1} \partial\left(\frac{1}{k_{p}+1}\left(\prod_{i=0}^{p-1} L_{2 i}^{k_{i}}\right) L_{2 p}^{k_{p}+1}\left(\bigwedge_{j=1}^{q-1} L_{2 \ell_{j}+1}\right)\right)
$$

et si $p<\ell_{q}$, on a

$$
L=(-1)^{q-1} \partial\left(\left(\prod_{i=0}^{p} L_{2 i}^{k_{i}}\right) L_{2 \ell_{q}}\left(\bigwedge_{j=1}^{q-1} L_{2 \ell_{j}+1}\right)\right) .
$$

La cohomologie des graphes à lignes est donc toujours triviale.

Théorème 7.10 (La cohomologie de Chevalley des graphes vectoriels). La cohomologie de Chevalley des graphes vectoriels est donnée par les roues de longueur impaire. Plus précisément, pour tout $n$, une base de $H^{n}$ est donnée par

$$
\left\{R_{2 k_{1}+1} \wedge R_{2 k_{2}+1} \wedge \cdots \wedge R_{2 k_{p}+1}: k_{1}<k_{2}<\cdots<k_{p}, \sum_{i=1}^{p}\left(2 k_{i}+1\right)=n\right\} .
$$


Démonstration. D'après la Proposition 7.1, tout cocycle $\delta$ est cohomologue à un cocycle $\delta-\partial \beta$ dont le symbole ne contient que des graphes avec des sommets simples. On suppose maintenant que $\delta$ a cette propriété. Chaque graphe de ce symbole est donc une union de composantes connexes qui sont soit des roues simples de longueur impaire soit des lignes simples. Le nombre de lignes est d'ailleurs fixé, égal au nombre de $0^{-}$.

On définit un nouvel ordre sur les graphes de ce type en posant :

$$
O^{\prime}(\Delta)=\left(\ell_{1}, \ldots, \ell_{p}, r_{1}, \ldots, r_{q}\right)
$$

où les $\ell_{i}$ sont les longueurs des lignes et les $r_{j}$ celles des roues. On range ces symboles en posant $\ell_{i}>r_{j}$ pour tout $i$ et $j$, on range ces ordres de graphes par l'ordre lexicographique sur les symboles.

L'ordre de $\delta$ est

$$
\mathcal{O}^{\prime}(\delta)=\max \left\{\mathcal{O}^{\prime}(\Delta), \Delta \text { apparaissant dans } \sigma_{\delta}\right\}
$$

On a donc $\mathcal{O}^{\prime}(\delta)=\left(\ell_{1}, \ldots, \ell_{p}, r_{1}, \ldots, r_{q}\right)$ avec $\ell_{1} \geq \ell_{2} \geq \ldots$ et $r_{1}>r_{2}>\ldots$, les seuls $\ell_{i}$ répétés sont pairs, les $r_{j}$ sont tous impairs. Le nouveau symbole de $\delta$ est

$$
\sigma_{\delta}^{\prime}=\sum_{\substack{\Delta \\ O^{\prime}(\Delta)=O^{\prime}(\delta)}} a_{\Delta} \Delta .
$$

Supposons qu'il existe un indice $i$ tel que $\ell_{i}$ est pair et $\ell_{i}+1$ n'appartient pas à $\left\{\ell_{1}, \ldots, \ell_{i-1}\right\}$. Appelons $i_{0}$ le premier indice pour lequel ceci se produit.

Si $\Delta$ apparait dans $\delta$ mais pas dans $\sigma_{\delta}$, on a vu que :

$$
\mathcal{O}(\partial \Delta)<\mathcal{O}(\delta) \oplus 1^{+} \text {. }
$$

D'après les lemmes précédents, si $\Delta$ apparaît dans $\sigma_{\delta}$ mais pas dans $\sigma_{\delta}^{\prime}$, alors le nouvel ordre $O^{\prime}(\partial \Delta)$ est strictement plus petit que

$$
\mathcal{O}^{\prime}(\delta) \oplus\left[i_{0}\right]=(\ell_{1}, \ldots, \underbrace{\ell_{i_{0}}+1}_{\left(i_{0}\right)}, \ldots, \ell_{p}, r_{1}, \ldots, r_{q}) .
$$

En effet, pour calculer $\partial \Delta$, on procède à des éclatements soit dans les lignes soit dans les roues. On ne retient que les éclatements qui ne disparaissent pas par symétrisation.

Enfin si $\Delta$ apparait dans $\sigma_{\delta}$ et dans $\sigma_{\delta}^{\prime}$, le nouvel ordre de $\partial \Delta$ est toujours inférieur ou égal à $O^{\prime}(\delta) \oplus\left[i_{0}\right]$, car on utilise les lemmes précédents, en procédant à des éclatements successivement des sommets des lignes et des sommets des roues. 
On a donc avec nos notations habituelles :

$$
S\left(\sigma_{\delta}^{\prime}\right)=a\left(\prod_{\ell_{i} \text { pair }} L_{\ell_{i}}\right)\left(\bigwedge_{\ell_{i} \text { impair }} L_{\ell_{i}}\right) \wedge\left(\bigwedge_{j=1}^{p} R_{j}\right) \quad(a \in \mathbb{R} \backslash\{0\})
$$

et

$$
S\left(\sigma_{\partial \delta}^{\prime}\right)=a\left(\prod_{\substack{\ell_{i} \text { pair } \\ i \neq i_{0}}} L_{\ell_{i}}\right) L_{\ell_{i_{0}}+1} \wedge\left(\bigwedge_{\ell_{i} \text { impair }} L_{\ell_{i}}\right) \wedge\left(\bigwedge_{j=1}^{p} R_{j}\right) \neq 0 .
$$

Ceci est donc impossible car $\delta$ est un cocycle, alors, il n'existe pas de $i$ tel que $\ell_{i}$ soit pair et $\ell_{i}+1$ n'appartient pas à $\left\{\ell_{1}, \ldots, \ell_{i-1}\right\}$.

On en déduit en particulier que s'il y a des lignes, alors, $\ell_{1}$ est impair et en retranchant à $\delta$ le cobord de

$$
\beta=a\left(\prod_{\ell_{i} \text { pair }} L_{\ell_{i}}\right) L_{\ell_{1}-1}\left(\bigwedge_{\ell_{i} \text { impair }}^{i>1} L_{\ell_{i}}\right) \wedge\left(\bigwedge_{j=1}^{p} R_{j}\right)
$$

on obtient un graphe $\delta-\partial \beta$ dont le nouvel ordre est strictement plus petit que celui de $\delta$. En répétant cette opération, on se ramène au cas où le symbole de $\delta$ ne contient que des graphes à roues impaires. Alors $S\left(\sigma_{\delta}\right)$ est un cocycle.

On recommence cette opération jusqu'à annulation de l'ordre des cocycles construits. On obtient :

$$
\delta=\partial \beta+\sum_{r_{1}, \ldots, r_{q}} a_{r_{1} \ldots r_{q}} R_{r_{1}} \wedge \cdots \wedge R_{r_{q}} .
$$

La cohomologie est donc engendrée par les graphes à roues de longueurs impaires. On a vu que ces graphes sont linéairement indépendants.

\section{Conclusion}

De Wilde et Lecomte [1983] on montré que la cohomologie différentielle de Chevalley de l'algèbre de Lie des champs de vecteurs $\mathscr{X}\left(\mathbb{R}^{d}\right)$ associée à la dérivée de Lie des fonctions $C^{\infty}$ sur $\mathbb{R}^{d}$ est l'algèbre $\bigwedge_{\text {inv }}\left(\mathfrak{g l}\left(\mathbb{R}^{d}\right)\right)$. La cohomologie est donc engendrée par les $n$-cochaînes $\theta^{(n)}\left(\xi_{1}, \ldots, \xi_{n}\right)=\mathfrak{a}\left(\operatorname{tr}\left(\operatorname{Jac}\left(\xi_{1}\right), \ldots, \operatorname{Jac}\left(\xi_{n}\right)\right)\right)$, pour $n$ impair, $n<2 d$.

Comme les cochaînes différentielles de De Wilde-Lecomte sont inductives, on en conclut immédiatement que la cohomologie différentielle correspondante sur $\mathbb{R}^{\infty}$ est l'algèbre extérieure engendrée par les $\theta^{(n)}, n$ impair quelconque.

Dans cet article, nous montrons que la cohomologie des graphes vectoriels qui coïncide avec la cohomologie de Chevalley sur $\mathbb{R}^{\infty}$ des cochaînes associées à une combinaison $\delta$ de graphes est engendrée par les roues impaires. 
Considérons le graphe à roue impaire de $n$ sommets

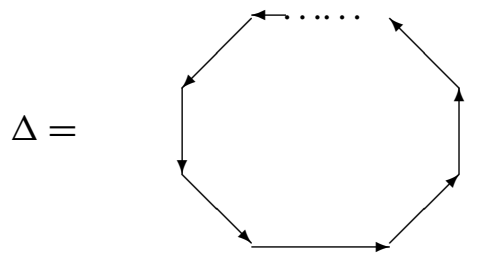

Notons $R_{n}=S(\Delta)$ la symétrisation de $\Delta$. Un calcul direct nous donne, pour tout $d$ et tout $\xi_{1}, \ldots, \xi_{n} \in \operatorname{Vect}\left(\mathbb{R}^{d}\right)$,

$$
\begin{aligned}
C_{R_{n}}\left(\xi_{1}, \ldots, \xi_{n}\right) & =\frac{1}{n !} \sum_{\sigma \in S_{n}} \varepsilon(\sigma) \sum_{1 \leq t_{1}, \ldots, t_{n} \leq d} \partial_{t_{n}} \xi_{\sigma(1)}^{t_{1}} \partial_{t_{1}} \xi_{\sigma(2)}^{t_{2}} \ldots \partial_{t_{n-1}} \xi_{\sigma(n)}^{t_{n}} \\
& =\theta^{(n)}\left(\xi_{1}, \ldots, \xi_{n}\right) .
\end{aligned}
$$

Proposition 8.1 (Les cohomologies différentielles et associées aux graphes coïncident). La cohomologie de Chevalley des cochaînes différentielles sur $\mathscr{X}\left(\mathbb{R}^{\infty}\right)$, à valeurs dans $T_{\text {poly }}^{0}\left(\mathbb{R}^{\infty}\right)$ coïncide avec la cohomologie de Chevalley des cochaînes associées à des combinaisons linéaires symétriques de graphes vectoriels, à valeurs dans $T_{\text {poly }}\left(\mathbb{R}^{\infty}\right)$.

\section{Références}

[Arnal et Masmoudi 2002] D. Arnal et M. Masmoudi, "Cohomologie de Hochschild des graphes de Kontsevich”, Bull. Soc. Math. France 130 :1 (2002), 49-69. MR 2003k :53122 Zbl 1028.46099

[Arnal et al. 2002] D. Arnal, D. Manchon et M. Masmoudi, "Choix des signes pour la formalité de M. Kontsevich”, Pacific J. Math. 203 :1 (2002), 23-66. MR 2003k :53123 Zbl 1055.53066

[Arnal et al. 2005] D. Arnal, A. Gammella et M. Masmoudi, "Chevalley cohomology for Kontsevich's graphs”, Pacific J. Math. 218 :2 (2005), 201-239. MR MR2218346 Zbl 05040421

[Berger 1982] R. Berger, "Cohomologie différentiable des algèbres de polynômes, de leurs localisées ou de leurs complétées, et des variétés”, Publ. Dép. Math. (Lyon) (N.S.) 5/A (1982), 1-19. MR 84k :16031 Zbl 0529.13004

[De Wilde et Lecomte 1983] M. De Wilde et P. B. A. Lecomte, "Cohomology of the Lie algebra of smooth vector fields of a manifold, associated to the Lie derivative of smooth forms", J. Math. Pures Appl. (9) 62 :2 (1983), 197-214. MR 85j :17017 Zbl 0481.58032

[Gammella et Halbout 2003] A. Gammella et G. Halbout, " $G_{\infty}$-formality theorem in terms of graphs and associated Chevalley-Eilenberg-Harrison cohomology", preprint 2003-015, Institut de Recherche Mathématique Avancée, Strasbourg, 2003.

[Gelfand et Fuks 1970] I. M. Gelfand et D. B. Fuks, "Cohomologies of the Lie algebra of tangential vector fields of a smooth manifold, II”, Funkcional. Anal. i Priložen. 4 :2 (1970), 23-31. In Russian ; translated in Funct. Anal. Appl. 4 (1970), 110-116. MR 44 \#2248 Zbl 0208.51401

[Kontsevich 2003] M. Kontsevich, "Deformation quantization of Poisson manifolds", Lett. Math. Phys. 66 :3 (2003), 157-216. MR 2005i :53122 Zbl 1058.53065 
[Vey 1975] J. Vey, "Déformation du crochet de Poisson sur une variété symplectique", Comment. Math. Helv. 50 :4 (1975), 421-454. MR 54 \#8765 Zbl 0351.53029

Received July 4, 2005.

WALID ALOULOU

DÉPARTEMENT DE MATHÉMATIQUES

Unité DE RECHERChe PhysiQue MATHÉmATIQUe

FACUlTÉ DES SCIENCES DE MONASTIR

AVENUE DE L'ENVIRONNEMENT

5019 MONASTIR

TUNISIE

Walid.Aloulou@ipeim.rnu.tn

DIDIER ARNAL

INSTITUT DE MATHÉMATIQUES DE BOURGOGNE

UMR CNRS 5584

UNIVERSITÉ DE BOURGOGNE

U.F.R. SCIENCES ET TECHNIQUES B.P. 47870

F-21078 DiJON CEDEX

FRANCE

Didier.Arnal@u-bourgogne.fr

\section{RIDHA CHATBOURI}

DÉPARTEMENT DE MATHÉMATIQUeS

Unité DE Recherche Physique Mathématique

FACULTÉ DES SCIENCES DE MONASTIR

AVENUE DE L'ENVIRONNEMENT

5019 MONASTIR

TUNISIE

Ridha.Chatbouri@ipeim.rnu.tn 